\title{
New dualities and misleading anomaly matchings from outer-automorphism twists
}

\author{
Sridip Pal and Jaewon Song \\ Department of Physics, University of California, San Diego, \\ La Jolla, CA 92093, U.S.A. \\ E-mail: srpal@ucsd.edu, jsong@physics.ucsd.edu
}

ABSTRACT: We study four-dimensional $\mathcal{N}=1,2$ superconformal theories in class $\mathcal{S}$ obtained by compactifying the $6 \mathrm{~d} \mathcal{N}=(2,0)$ theory on a Riemann surface $\mathcal{C}$ with outerautomorphism twist lines. From the pair-of-pants decompositions of $\mathcal{C}$, we find various dual descriptions for the same theory having distinct gauge groups. We show that the various configurations of the twist line give rise to dual descriptions for the identical theory. We compute the 't Hooft anomaly coefficients and the superconformal indices to test dualities. Surprisingly, we find that the class $\mathcal{S}$ theories with twist lines wrapping 1-cycles of $\mathcal{C}$ have the identical 't Hooft anomalies as the ones without the twist line, whereas the superconformal indices differ. This provides a large set of examples where the anomaly matching is insufficient to test dualities.

Keywords: Anomalies in Field and String Theories, Supersymmetric Gauge Theory, Supersymmetry and Duality, M-Theory

ARXIV EPRINT: 1611.00694 


\section{Contents}

1 Introduction 1

2 New dualities from the outer-automorphism twist 3

$2.1 \mathcal{N}=2$ dualities 3

2.2 Adding punctures 8

$\begin{array}{lr}2.3 \mathcal{N}=1 \text { dualities } & 10\end{array}$

3 Misleading anomalies and the superconformal indices $\quad 13$

3.1 Central charges of the $\mathcal{N}=2$ theories $\quad 13$

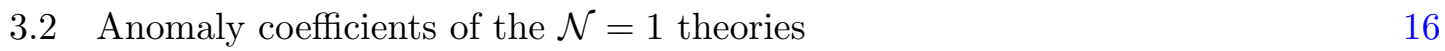

$\begin{array}{lll}3.3 & a \text {-maximization } & 18\end{array}$

$\begin{array}{ll}3.4 & \text { Superconformal index } \\ \end{array}$

4 Conclusion 23

\section{Introduction}

One of the most important discoveries in supersymmetric field theory is that there can be two different theories flowing to the same infrared (IR) fixed point [1]. This IR duality has extensively been studied for the last two decades. However, the test of the duality is rather difficult in general since at least one of the dual descriptions is strongly-coupled in the IR. The usual test involves quantities that can be computed reliably due to the holomorphic nature: such as matching of chiral operators, moduli space of vacua and the 't Hooft anomalies. One of the most useful quantities to compute is the 't Hooft anomaly coefficients as they are invariant under the renormalization group (RG) flow, and hence can be computed in the weakly coupled regime. Thus, two very different gauge theories having the same anomaly coefficients is a quantitative check of the duality.

The two different theories having the same 't Hooft anomalies, in spite of being a strong check, does not necessarily imply that two theories are dual to each other. After all, they are a small set of numbers that one can compute from the theory so that the matching can be a coincidence. Indeed such example was given in $[2,3]$. But such examples are rather difficult to find because it involves an understanding of strong-coupling physics at the fixed point.

Recent developments in the supersymmetric localization provide us with new tools for testing dualities. This gives a new set of physical observables, e.g. supersymmetric partition functions, that can reliably be computed. These supersymmetric partition functions allow us to extract certain operator spectrum of the fixed point, and also provide more refined checks for the dualities. See the recent review [4] and the references therein for more details. So it is a natural question to ask whether there can be a pair of theories with the 
same 't Hooft anomalies but different supersymmetric partition functions. Indeed in the paper [3], supersymmetric partition function was used to distinguish the IR behavior not captured by the anomalies.

In this paper, we present a large set of examples that have the same anomaly coefficients but different supersymmetric partition functions (namely the superconformal index). This set of examples arises from the so-called class $\mathcal{S}$ theories $[5,6]$, coming from the $6 \mathrm{~d}$ $\mathcal{N}=(2,0)$ theory compactified on a Riemann surface $\mathcal{C}_{g, n}$ of genus $g$ with $n$ punctures. The Riemann surface is called the UV curve, and one can get $\mathcal{N}=1$ or $\mathcal{N}=2$ theory in fourdimension depending on the choice of partial topological twist along the UV curve. One of the powerful features of the class $\mathcal{S}$ theory is that various properties become manifest through the underlying geometry of $\mathcal{C}_{g, n}$. Especially, different pair-of-pants decompositions give various dual descriptions. In this way, new $\mathcal{N}=2$ S-dualities $[5,7-17]$ and $\mathcal{N}=1$ dualities have been found [18-25].

The setup we consider in this paper is the class $\mathcal{S}$ theories of type $\Gamma=A D E$ obtained by $6 \mathrm{~d} \mathcal{N}=(2,0)$ theory of type $\Gamma$ wrapped on a UV curve $\mathcal{C}_{g, n}$ of genus $g$ and $n$ punctures. Let us denote the $4 \mathrm{~d}$ theory obtained in this way as $\mathcal{T}\left[\mathcal{C}_{g, n}\right] .{ }^{1}$ When the group $\Gamma$ admits an outer-automorphism action, one can introduce an outer-automorphism twist line, or twisted punctures $[8,12,13,16,17,23] .{ }^{2}$ This twisting can be understood as introducing an orientifold plane in the type IIA intersecting brane description, which changes the gauge group and the matter content of the $4 \mathrm{~d}$ theory appropriately. The twist lines can emanate from a twisted puncture and end on another twisted puncture. The twist line can also form a loop wrapping various 1-cycles of $\mathcal{C}_{g, n}$ [11]. Let us call the latter ones as the twist loops. One can have various configurations of the twist loop for a given UV curve. Surprisingly, it turns out that various configurations for the twist lines can be deformed to each other so that we find:

there is only one physically inequivalent configuration for the $\mathbb{Z}_{2}$ twist loop.

Different topological configurations give rise to dual descriptions of the same theory upon pair-of-pants decomposition. ${ }^{3}$

Also, when there is a twisted-puncture on the UV curve, we find that adding a twist loop to the curve does not change the theory but gives a dual description:

$$
\mathcal{T}\left[\mathcal{C}_{g, n}\right] \simeq \mathcal{T}\left[\tilde{\mathcal{C}}_{g, n}\right] \quad \text { (if any of the punctures are twisted) }
$$

where $\tilde{\mathcal{C}}_{g, n}$ denotes the same UV curve as $\mathcal{C}_{g, n}$ except for a twist line wrapping around 1-cycle. It is rather surprising at first sight, but it turns out the two configurations can be obtained by deforming the geometry in a smooth way.

We compute the anomaly coefficients and the superconformal indices to test the dualities. Since the theories that we discuss in this paper are mostly 'non-Lagrangian', we rely

\footnotetext{
${ }^{1}$ Here we suppress the reference to the type $\Gamma$ of the $6 \mathrm{~d} \mathcal{N}=(2,0)$ theory and the type of each puncture.

${ }^{2}$ We only discuss the $\mathbb{Z}_{2}$-twist in this paper. When $\Gamma=D_{4}$, one can also twist with the $\mathbb{Z}_{3}$ action. Most of the story regarding the anomalies vs indices hold for this case as well.

${ }^{3}$ The twist lines define a $\mathbb{Z}_{2}$-bundle over the UV curve $\mathcal{C}$. The topological class is captured by its StiefelWhitney class, which takes value in $H^{2}\left(\mathcal{C}, \mathbb{Z}_{2}\right)=\mathbb{Z}_{2}$. This is consistent with what we find. We would like to thank Yuji Tachikawa for pointing this out to us.
} 
on the $4 \mathrm{~d} / 2 \mathrm{~d}$ correspondence of indices for the class $\mathcal{S}$ theories [26-32]. We find that the anomalies for the class $\mathcal{S}$ theories do not depend on the existence of the twist loop, but the theories belong to different universality classes, provided none of the punctures is twisted:

$$
\mathcal{A}\left(\mathcal{T}\left[\mathcal{C}_{g, n}\right]\right)=\mathcal{A}\left(\mathcal{T}\left[\tilde{\mathcal{C}}_{g, n}\right]\right) \quad \text { whereas } \quad \mathcal{T}\left[\mathcal{C}_{g, n}\right] \neq \mathcal{T}\left[\tilde{\mathcal{C}}_{g, n}\right]
$$

where $\mathcal{A}(\cdot)$ denotes the anomaly coefficients of the theory. Even though the anomalies match, these two theories differ, which can be checked from distinct superconformal indices. If we have at least one twisted puncture, we obtain the new dualities as in (1.1).

The organization of this paper is as follows. In section 2, we describe the new dualities we obtain by considering class $\mathcal{S}$ theories with outer-automorphism twist loops on the UV curve. In section 3, we compute the 't Hooft anomaly coefficients and the superconformal indices to test the dualities. We find that the anomaly coefficients do not get altered by the presence of loop of the twist line, but the index gets modified. Then we conclude with some remarks.

\section{New dualities from the outer-automorphism twist}

\section{$2.1 \mathcal{N}=2$ dualities}

$\mathcal{N}=\mathbf{2}$ theories of class $\mathcal{S}$. Let us review aspects of the class $\mathcal{S}$ theories [5,6] that we need in our discussion. The class $\mathcal{S}$ theories arise from wrapping $6 \mathrm{~d} \mathcal{N}=(2,0)$ theory of type $\Gamma \in A D E$ on a Riemann surface $\mathcal{C}$ with or without appropriate punctures. The punctures arise from various codimension 2 defects of the $6 \mathrm{~d}$ theory. In order to preserve some amount of supersymmetry, one should perform partial topological twist along the direction of $\mathcal{C}$. Depending on the choice of embedding $\mathrm{SO}(2)$ holonomy group into the $\mathrm{SO}(5)_{R}$ of the 6d theory, we get either $\mathcal{N}=1$ or $\mathcal{N}=2$ supersymmetry in four-dimensions [19, 21]. Let us first focus on $\mathcal{N}=2$ case for the moment.

The field theory represented by the UV curve is obtained by considering a pair-ofpants decomposition of $\mathcal{C}$, which is the building block for any (negatively curved) Riemann surface. To each pair-of-pants or the 3-punctured sphere, one associate the $T_{\Gamma}$ theory, which is an $\mathcal{N}=2$ superconformal theory with $\Gamma \times \Gamma \times \Gamma$ flavor symmetry. See [33] and the references therein for more detailed information about this theory. To each puncture ${ }^{4}$ of the 3-punctured sphere, we associate a flavor symmetry $\Gamma$. When $\Gamma$ admits a $\mathbb{Z}_{2}$ outerautomorphism, ${ }^{5}$ one can also consider a variation of the $T_{\Gamma}$ theory, which we call $\tilde{T}_{\Gamma}[7,8$, $12,13,16,17,23]$. It is also an $\mathcal{N}=2 \mathrm{SCFT}$, but with the flavor symmetry $\Gamma \times G \times G$, where $G$ is the group associated to the $\Gamma$ by 'folding' the associated Dynkin diagram with respect to the outer-automorphism action. Here, $G$ associated to $\Gamma$ is given in table 1 . In this case, two of the punctures in a pair-of-pants become 'twisted', and carry the flavor symmetry $G$. The basic building blocks are drawn in figure 1 .

A Riemann surface $\mathcal{C}$ can be obtained by gluing a number of pair-of-pants (or 3punctured sphere). The gluing operation is translated to the gauging of the flavor symmetry associated with the punctures. Since a Riemann surface admits various pair-of-pants

\footnotetext{
${ }^{4}$ They are maximal punctures. Non-maximal puncture can be obtained via certain nilpotent Higgsing.

${ }^{5}$ We only consider $\mathbb{Z}_{2}$ outer-automorphism. When $\Gamma=D_{4}$, one can also consider the $\mathbb{Z}_{3}$ twist.
} 


\begin{tabular}{|c|cccc|}
\hline$\Gamma$ & $A_{2 n}$ & $A_{2 n-1}$ & $D_{n+1}$ & $E_{6}$ \\
\hline$G$ & $C_{n}$ & $B_{n}$ & $C_{n}$ & $F_{4}$ \\
\hline
\end{tabular}

Table 1. $\mathbb{Z}_{2}$ Outer-automorphisms of the $A D E$ group. Here $G$ is the group obtained from folding the Dynkin diagram of $\Gamma$.

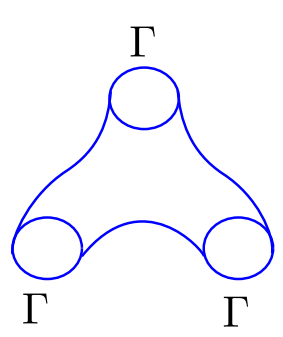

(a) A pair-of-pants: $T_{\Gamma}$.
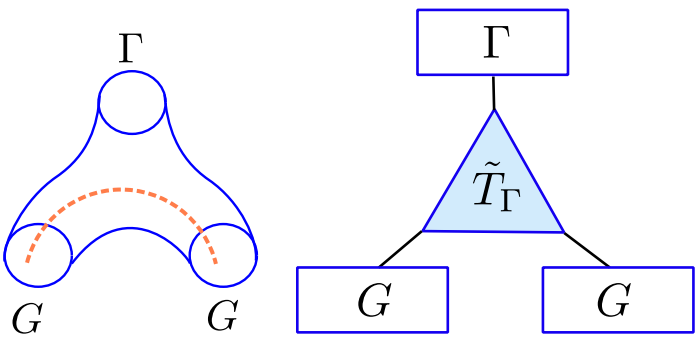

(b) A pair-of-pants with a twist line: $\tilde{T}_{\Gamma}$.

Figure 1. Two basic building blocks; $T_{\Gamma}$ and $\tilde{T}_{\Gamma}$. The twist line (orange) connects between the two twisted punctures with the flavor symmetry $G$.

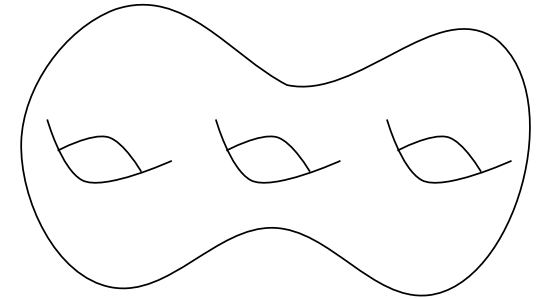

(a) $\mathcal{C}_{3,0}$ : UV curve without twist loop.

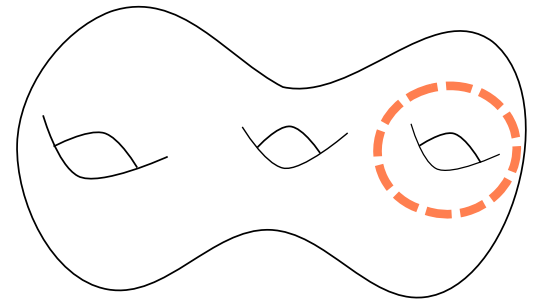

(b) $\tilde{\mathcal{C}}_{3,0}:$ UV curve with a twist loop.

Figure 2. The twist line (orange) wraps around one hole. The diagram depicts the case $g=3$.

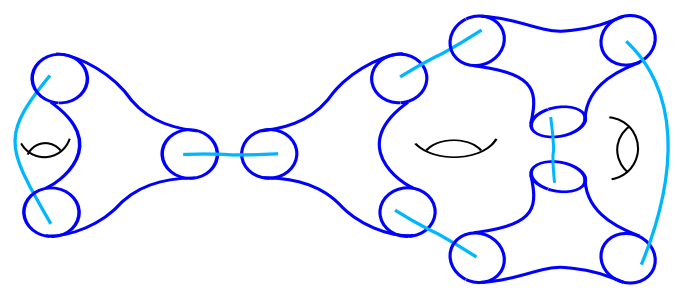

(a) A pair-of-pants decomposition of $\mathcal{C}_{3,0}$.

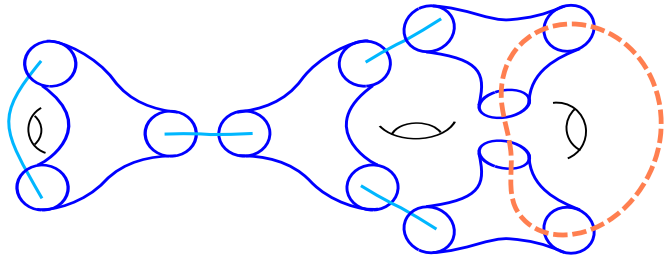

(b) A pair-of-pants decomposition of $\tilde{\mathcal{C}}_{3,0}$.

Figure 3. Pair-of-pants decompositions of $\mathcal{C}_{3,0}$ and $\tilde{\mathcal{C}}_{3,0}$. The blue line represents $\Gamma$ gluing. The orange line represents the $\mathbb{Z}_{2}$-twist line, which up on gluing we get the gauge group $G$.

decompositions, we have one-to-many correspondence. The different choices give the dual descriptions for the same theory. See figure 2 and 3.

Warm up: $\boldsymbol{g}=\mathbf{2}$ duality. Let us first consider the case of the UV curve $\tilde{\mathcal{C}}_{2,0}$ of genus 2 with a twist line wrapping around a hole. We can decompose the UV curve in terms of the pairs-of-pants in two distinct ways as given in the 1.h.s. of figure 4 . 


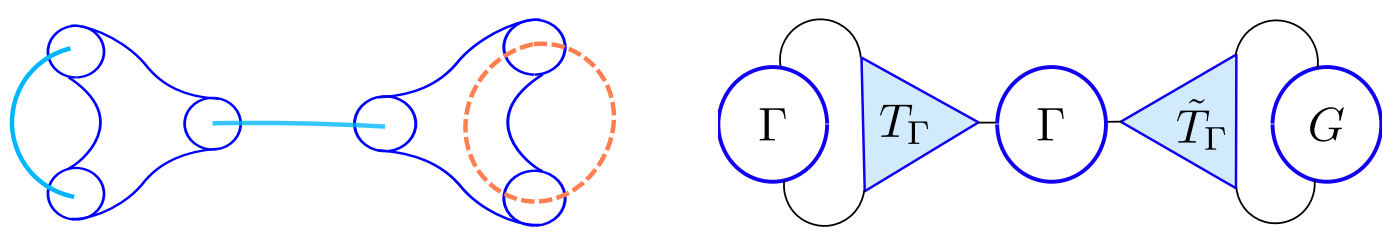

(a) A pair-of-pants decomposition.

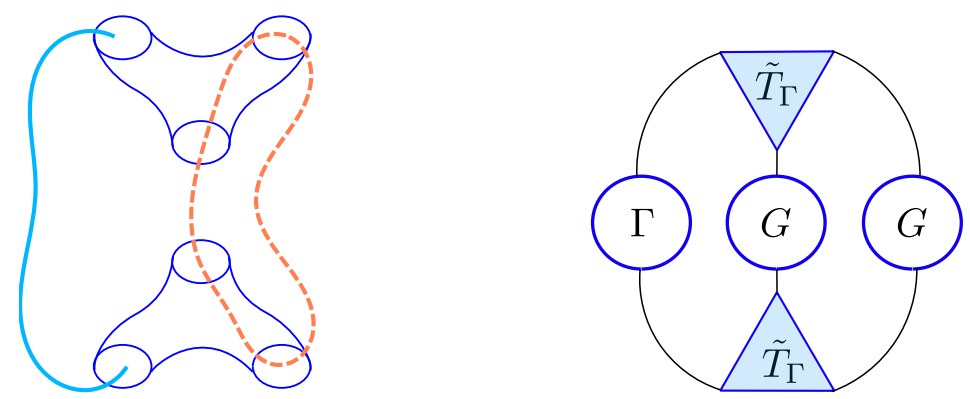

(b) Another pair-of-pants decomposition.

Figure 4. Two ways of decomposing the UV curve $\tilde{\mathcal{C}}_{2,0}$ (labelled as $a$ and $b$ ). Orange line is the twist line. Blue lines represent gluing with $\Gamma$ group. Orange line represent gluing with the $G$ (twisted) gauge group.

These two different pair-of-pants decompositions will lead to two dual descriptions as given in the r.h.s. of figure 4 . The first one will yield a $T_{\Gamma}$ block and a $\tilde{T}_{\Gamma}$ block with $\Gamma \times \Gamma \times G$ gauge symmetry, while the second decomposition will yield two $\tilde{T}_{\Gamma}$ blocks with $\Gamma \times G \times G$ gauge symmetry. Because they come from the same Riemann surface, the two pair-of-pants decompositions should describe the same physics. This particular duality can be thought of as a corollary of the basic S-duality for the 4-punctured sphere theory (analogous to the crossing symmetry) formed by gluing $T_{\Gamma}$ or $\tilde{T}_{\Gamma}[5,7,23]$. We can form the genus 2 surface by gluing the punctures, which is equivalent to gauging the corresponding flavor symmetry. In this way, we obtain the two configurations as in figure 4. As we will show in section 3.1 and 3.4, the central charges and the superconformal indices for the two dual frames are identical.

Now, let us consider the situation where the twist line wraps around the two holes at the same time as in figure 5. Let us call this UV curve as $\tilde{\mathcal{C}}_{2,0}^{\prime}$. Note that $\tilde{\mathcal{C}}_{2,0}$ and $\tilde{\mathcal{C}}_{2,0}^{\prime}$ are topologically distinct since the twist line cannot be continuously deformed to the other shape. As before, consider a pair-of-pants of decomposition. We get two $\tilde{T}_{\Gamma}$ blocks with $\Gamma \times G \times G$ symmetry, the same dual frame as we obtained from the second pair-of-pants decomposition of $\tilde{\mathcal{C}}_{2,0}$ in figure 4 . They are the same since there is a permutation symmetry among the punctures for the $T_{\Gamma}$ and $\tilde{T}_{\Gamma}$ theory.

Another dual frame is given as in figure 6. As we can see from the right-hand-side of the figure, this configuration can be obtained from taking the basic S-dual (crossing symmetry) of the middle node of figure 5. This can be thought of as having two twist loops wrapping each hole, leading us to a different topology of the twist line configuration. Therefore, we conclude that there is only one physically inequivalent configuration of the 


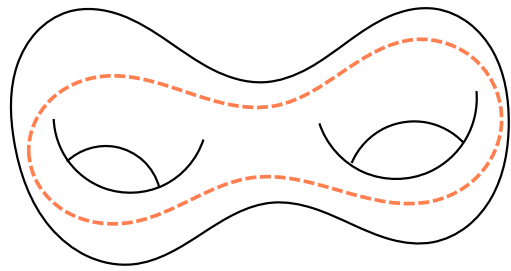

(a) The UV curve $\tilde{\mathcal{C}}_{2,0}^{\prime}$.

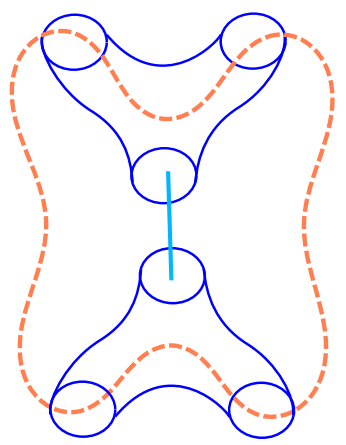

(b) A pair-of-pants decomposition.

Figure 5. The UV curve $\tilde{\mathcal{C}}_{2,0}^{\prime}$. The twist line (orange) wraps around two holes. The pair-of-pants decomposition reveals that we get the same theory as the case where the twist line wraps only one hole.

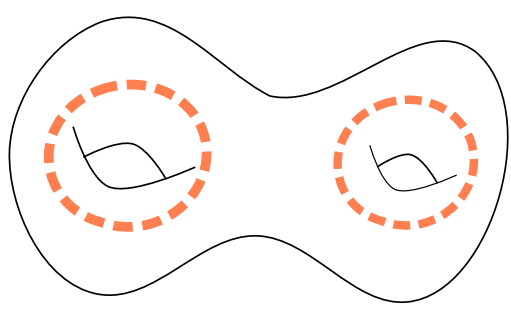

(a) The UV curve $\tilde{\mathcal{C}}_{2,0}^{\prime \prime}$.

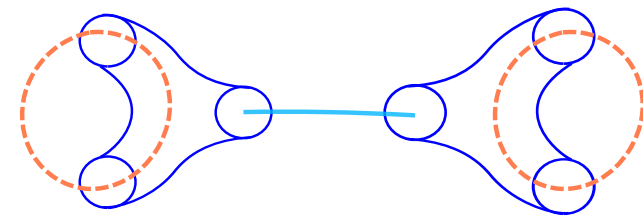

(b) A pair-of-pants decomposition.

Figure 6. The UV curve $\tilde{\mathcal{C}}_{2,0}^{\prime \prime}$ and its pair-of-pants decomposition. There are two separate twist loops wrapping each hole.

twist line in a sense that all the configurations are related by a chain of dualities.

S-dualities in the presence of the twist lines. Now, let us show in general that the different configurations for the twist lines are dual to one another. The configurations for the twist loops are related to one another by the following operations:

1. swapping the 1-cycles as in figure 7 .

2. one twist line wrapping two cycles can be replaced by the one twist line wrapping one hole as in figure 8 .

All the other configurations for the twist loops can eventually be mapped to the one wrapping a single 1-cycle upon performing a sequence of two operations. We see that the first operation of swapping the holes can be done by deforming the geometry in a continuous way. Therefore the two configuration belongs to the same conformal manifold. So there is no preferred choice of pair-of-pants decomposition among the two. In other words, one can obtain two different pair-of-pants decompositions related by duality.

The second operation can be justified by looking at the pair-of-pants decompositions. There is a dual frame where we obtain the same theory upon flipping the pairs-of-pants. One can obtain the other side of the twist line configuration by 1) decomposing into the 

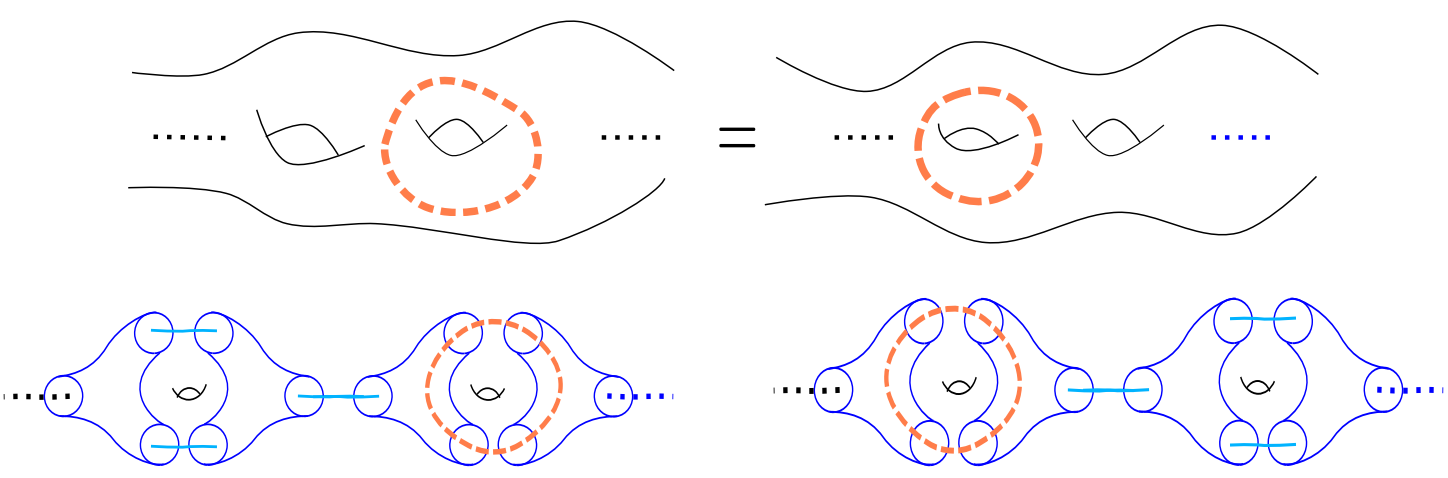

Figure 7. Swapping holes and the pair-of-pants decompositions of corresponding configurations.
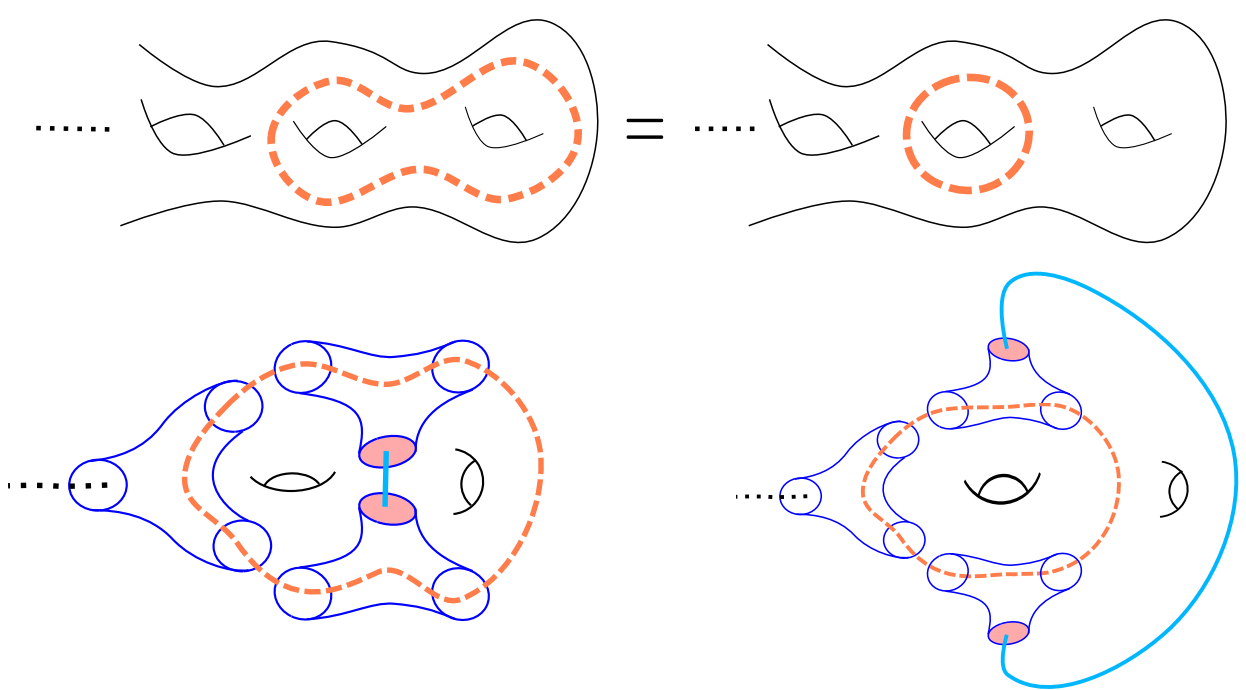

Figure 8. Upon decomposing the left configuration and gluing back upon flipping the pairs-ofpants, we obtain the configuration on the right, where twist loop wraps only one 1-cycle.

pair-of-pants, 2) rotating some of the pairs-of-pants, 3) glue them back in such a way that the twist line wraps around only one cycle. See figure 8. Therefore, for the UV curve with some configuration of twist loops, we can always pick a dual frame which involves only one twist loop wrapping one 1-cycle on the curve.

Notice that the first operation can be obtained by successive applications of the second operation. First, start with the twist loop wrapping one hole. It is now identical to the configuration where single twist line wrapping two neighboring holes. Finally, one can 'shrink' the loop by choosing the other hole.

Let us point out that the equivalence of the configurations in figure 9 can also be obtained by performing duality (crossing symmetry) for the basic block of the 4-punctured sphere. Let us note that from the perspective of the definition of the twist line on the curve, the equivalence is a simple fact coming from the property of the branch cuts. But from the field theory perspective, this equivalence leads to the duality. 

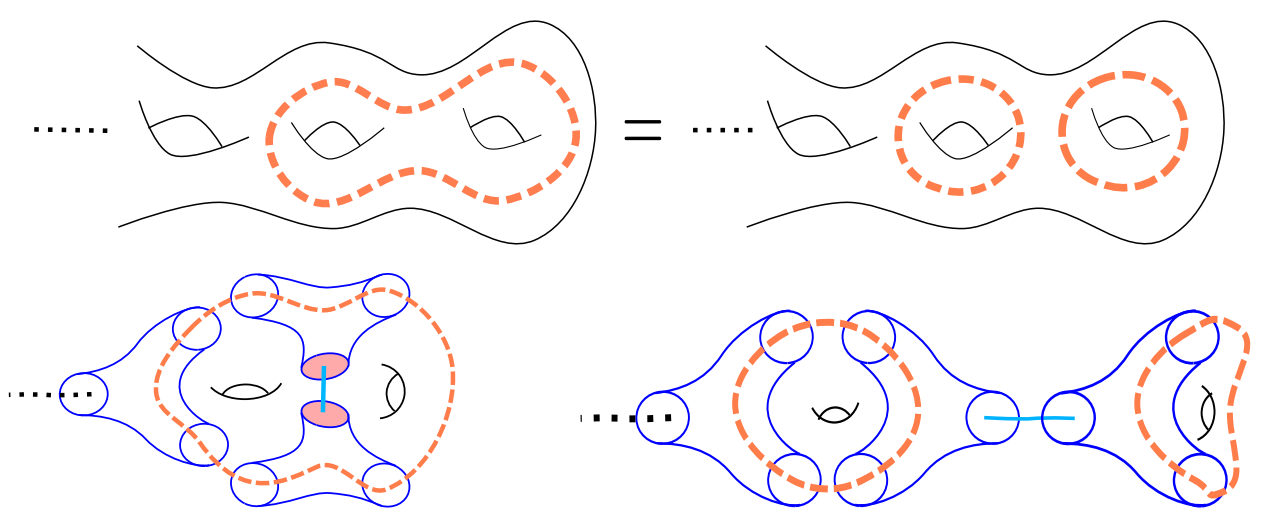

Figure 9. Joining two twist loops to obtain one loop wrapping around two holes and pair-of-pants decompositions of two configurations.

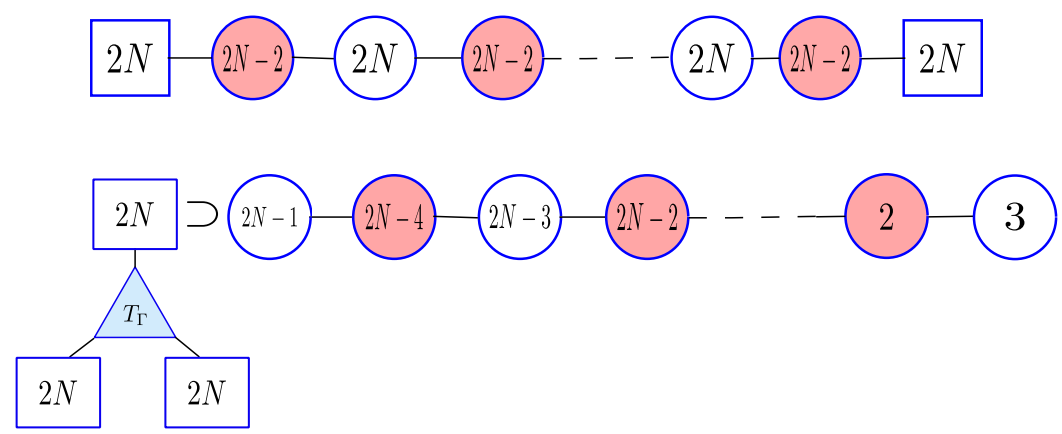

Figure 10. Theory A: a linear quiver with $\mathrm{SO}(2 N) \times \mathrm{SO}(2 N)$ flavor symmetry and its dual where we have $T_{\mathrm{SO}(2 N)}$ with a superconformal tail. The shaded nodes are USp groups.

\subsection{Adding punctures}

Until now, we have been considering the UV curve without a puncture i.e $\mathcal{C}_{g, 0}$. When there is no puncture, the theory has no non-R global symmetry (for the $\mathcal{N}=1$ case we discuss later in this section, there is only $\mathrm{U}(1)$ non- $\mathrm{R}$ symmetry). By adding punctures to the UV curve, we obtain theories with larger global symmetries.

Let us consider a linear quiver theory, which can be obtained by a sphere with a number of punctures. It is known [23] that linear SO-USp quiver has a dual frame that yields $T_{\mathrm{SO}(2 N)}$ or $\tilde{T}_{\mathrm{SO}(2 N)}$ block with the superconformal tail depending on whether the quiver has $\mathrm{SO}(2 N)$ or $\operatorname{USp}(2 N-2)$ flavor symmetry at the ends. The dualities are given as in figure 10 and figure 11. Let us call them theory A and B respectively.

Now in each of the dual frames, we gauge the flavor symmetry of $T_{\mathrm{SO}(2 N)}$ or $\tilde{T}_{\mathrm{SO}(2 N)}$ block to obtain the theory $C$ and $D$ respectively as in figure 12 . This amounts to gluing of the flavor symmetry of the linear quiver, which results in the formation of a circular quiver. Now, it is easy to see that upon gluing, both $A$ and $B$ yields the same circular quiver as in figure 13. This implies that theory $C$ and $D$ are dual to each other and also to the circular quiver.

We can explain this duality in terms of the UV curve and twist lines as in figure 14. The UV curve $\mathcal{C}_{1,6}$ corresponds to theory $C$, where we have $T_{\mathrm{SO}(2 N)}$ block with two of 


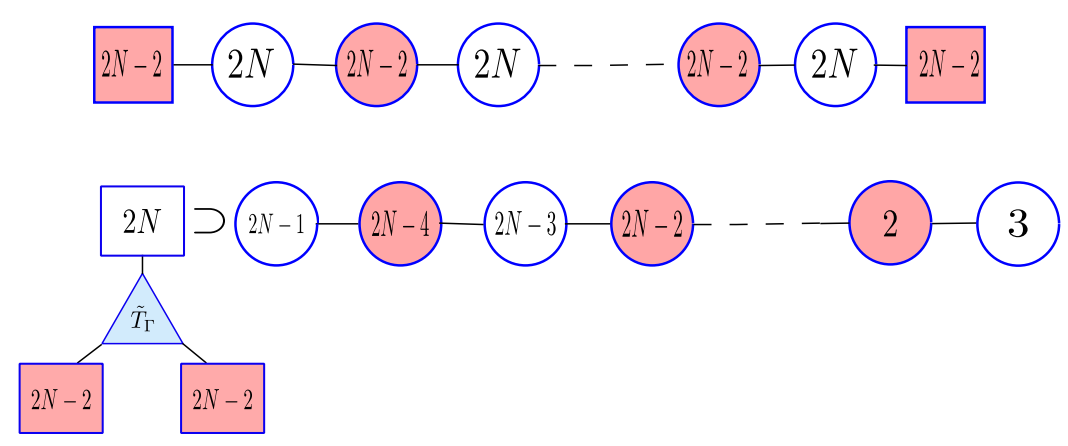

Figure 11. Theory B: a linear quiver with $\operatorname{USp}(2 N-2) \times \operatorname{USp}(2 N-2)$ flavor symmetry and its dual where we have $\tilde{T}_{\mathrm{SO}(2 N)}$ with a superconformal tail. The shaded nodes are USp groups.

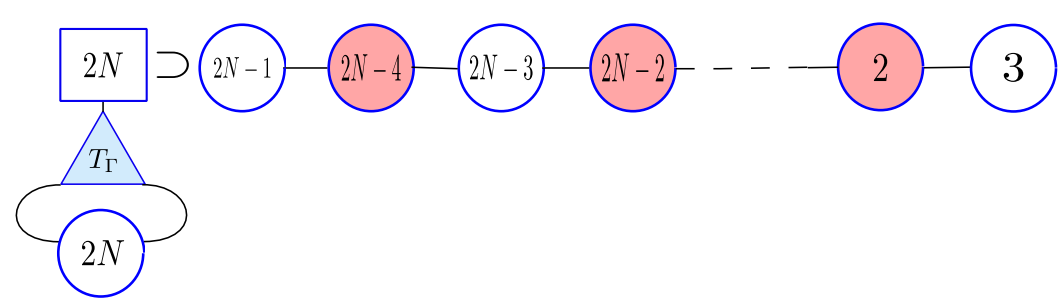

(a) Theory $\mathrm{C}$ formed by gluing the two ends of the quiver $\mathrm{A}$.

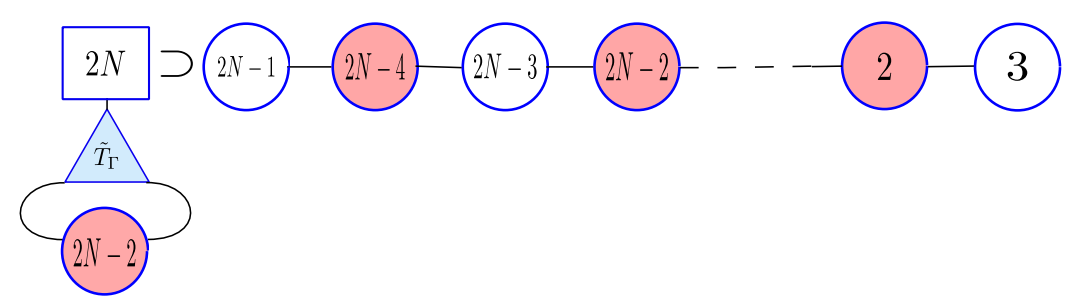

(b) Theory D formed by gluing the two ends of the quiver B.

Figure 12. Theories C and D obtained from theory A and B as in figure 10, 11 upon gluing flavor symmetry of $T_{\mathrm{SO}(2 N)}$ and $\tilde{T}_{\mathrm{SO}(2 N)}$ respectively. They are dual to the circular quiver of figure 13 . The shaded nodes denote the USp groups.

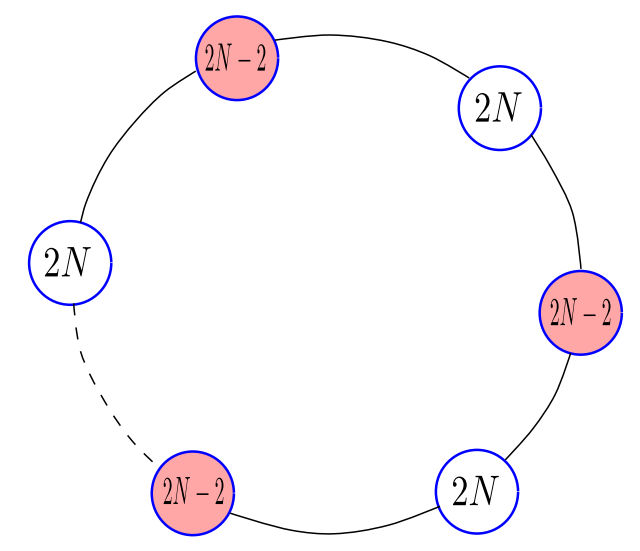

Figure 13. Circular SO-USp quiver. The shaded nodes are USp groups. 


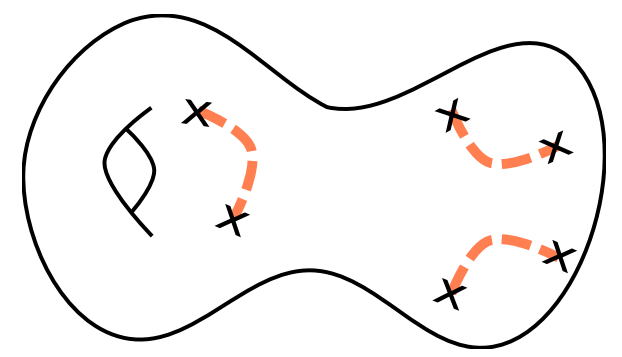

(a) The UV curve $\mathcal{C}_{1,6}$ with 6 twisted punctures. This corresponds to the theory $C$ as in figure 12 .

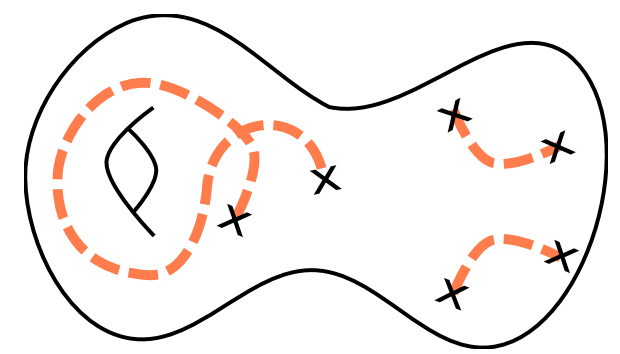

(c) The UV curve $\tilde{\mathcal{C}}_{1,6}$, where the twist line wraps the hole as the punctures cross each other without colliding.

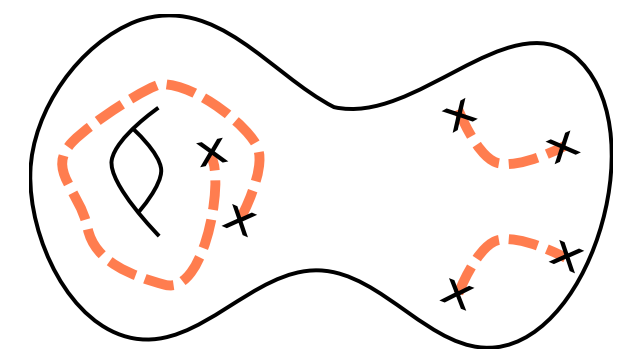

(b) Move the twisted punctures to wrap around the hole.

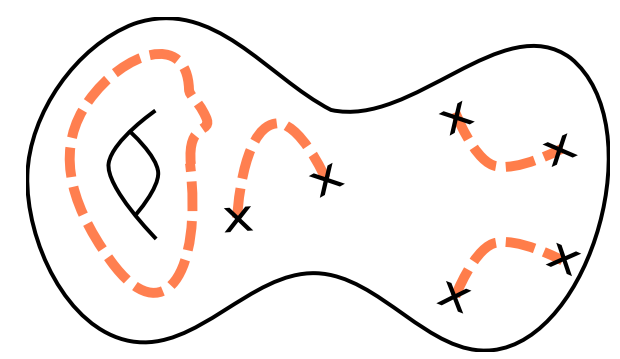

(d) Snip a part of the twist line, resulting the UV curve $\tilde{\mathcal{C}}_{1,6}$ with an extra twist loop. This corresponds to the theory $D$ in figure 12 .

Figure 14. Construction of the UV curve with a twist loop wrapping a hole from the UV curve without the twist loop.

its $S O$ flavor symmetry gauged, once the punctures are chosen to be the twisted null puncture. The UV curve $\tilde{\mathcal{C}}_{1,6}$ corresponds to theory $D$ with a $\tilde{T}_{\mathrm{SO}(2 N)}$ block and the USp flavor symmetry gauged.

Here, we see that the existence of the twist line wrapping around a loop in the UV curve does not change the theory, as long as there is a pair of the twisted punctures in the UV curve already. One can have this extra twist loop by moving the twisted punctures around as described in figure 14.

Let us note that for $\Gamma=A_{n}, E_{6}$ we do not have a linear quiver description as in the case of $D_{n}$. Nevertheless, the argument in terms of the UV curve and the twist line should go through. Therefore similar construction should yield new dualities for all $\Gamma=A_{n}, D_{n}, E_{6}$, regardless of the type of punctures as long as they are twisted. Therefore we arrive at the statement that

$$
\mathcal{T}\left[\mathcal{C}_{g, n}\right] \simeq \mathcal{T}\left[\tilde{\mathcal{C}}_{g, n}\right] \quad \text { (if any of the punctures is twisted) }
$$

where $\mathcal{C}_{g, n}$ and $\tilde{\mathcal{C}}_{g, n}$ are identical UV curves but the latter one has a twist loop.

\section{$2.3 \mathcal{N}=1$ dualities}

$\mathcal{N}=1$ class $\mathcal{S}$ theory. Let us generalize our discussion to the $\mathcal{N}=1$ class $\mathcal{S}$ theories [19-25]. In addition to the UV curve we choose, an $\mathcal{N}=1$ class $\mathcal{S}$ theory is specified 
by the choice of the normal bundles $\mathcal{L}(p) \oplus \mathcal{L}(q) \rightarrow \mathcal{C}_{g, n}$ over the UV curve. The two integers $(p, q)$ denote the degrees of the line bundles. In order to preserve any amount of supersymmetry, they have to satisfy $p+q=2 g-2+n$ for the UV curve having genus $g$ and $n$ punctures. When either $p$ or $q$ is set to zero, we obtain $\mathcal{N}=2$ theory. To each puncture, we also assign $\mathbb{Z}_{2}$-valued color $\sigma= \pm$. These theories admit $\mathrm{U}(1)_{+} \times \mathrm{U}(1)_{-}$ global symmetry generated by what we call $\left(J_{+}, J_{-}\right)$. It comes from a subgroup of $\mathrm{SO}(5)_{R}$ symmetry of the $6 \mathrm{~d} \mathcal{N}=(2,0)$ theory, which is preserved after the partial topological twist on the UV curve.

Similar to the $\mathcal{N}=2$ case, we consider pair-of-pants decompositions of $\mathcal{C}_{g, n}$ to obtain various dual descriptions of the SCFT. However, now to each pair-of-pants, we assign a color $\sigma= \pm .{ }^{6}$ The numbers of \pm -colored pairs-of-pants are given by the degrees of the line bundles $p$ and $q$ respectively. To each $\sigma$-colored pair-of-pants, we associate the $T_{\Gamma}$ theory if there is no twist line. If there is a twist line running through the pair-of-pants, we associate the $\tilde{T}_{\Gamma}$ instead. The color on the pair-of-pants does not change the theory $T_{\Gamma}$ or $\tilde{T}_{\Gamma}$ itself. Instead, it determines how the gluing should be done.

The gluing is done in the following manner: if $\sigma_{i}=\sigma_{j}$, the $i$-th and $j$-th pairs-of-pants are glued with the $\mathcal{N}=2$ vector multiplet. If $\sigma_{i} \neq \sigma_{j}$, they are glued with the $\mathcal{N}=1$ vector. When the gluing is done using $\mathcal{N}=2$ vector, we add the following superpotential

$$
W_{\mathcal{N}=2}=\operatorname{Tr} \phi\left(\mu_{i}+\mu_{j}\right)
$$

for each $\mathcal{N}=2$ node, where $\mu_{i}, \mu_{j}$ are the moment maps associated to the punctures and $\phi$ is the adjoint chiral multiplet in the $\mathcal{N}=2$ vector multiplet. For the $\mathcal{N}=1$ nodes, we add

$$
W_{\mathcal{N}=1}=\operatorname{Tr} \mu_{i} \mu_{j}
$$

instead. We preserve $\mathrm{U}(1)_{+} \times \mathrm{U}(1)_{-}$global symmetry upon this gluing, where the charges for the $\mu_{i}$ are $\left(J_{+}, J_{-}\right)=\left(1+\sigma_{i}, 1-\sigma_{i}\right)$ and for the $\phi$ are $\left(J_{+}, J_{-}\right)=\left(1-\sigma_{i}, 1+\sigma_{i}\right)$.

Finally, when the color of the puncture is identical to the pair-of-pants it belongs to, then we do nothing. But if the color of the puncture is opposite to that of the pair-of-pants, we add a chiral multiplet $M$ transforming under the adjoint of $\Gamma$ (or $G$ if the puncture is twisted) and couple via superpotential $W=\operatorname{Tr} M \mu$.

The symmetries $\mathrm{U}(1)_{+}$and $\mathrm{U}(1)_{-}$both are the candidate $R$-symmetry of the theory so that the superpotential should have the charge $\left(J_{+}, J_{-}\right)=(2,2)$. The superconformal $R$ symmetry can be written as

$$
R_{\mathrm{IR}}=\frac{1+\epsilon}{2} J_{+}+\frac{1-\epsilon}{2} J_{-}=R_{0}+\epsilon \mathcal{F},
$$

where $R_{0}=\frac{1}{2}\left(J_{+}+J_{-}\right)$and $\mathcal{F}=\frac{1}{2}\left(J_{+}-J_{-}\right)$. The value of $\epsilon$ is fixed through the $a$-maximization [34].

Dualities. Let us consider an example: choose the UV curve as $\mathcal{L}(1) \oplus \mathcal{L}(1) \rightarrow \tilde{\mathcal{C}}_{2,0}$ with the twist line wrapping one of the holes. Two pair-of-pants decompositions will

\footnotetext{
${ }^{6}$ Generally, it is labelled by $(p, q) \in \mathbb{Z}$ with $p+q=1$. The choice \pm refers to $(p, q)=(1,0)$ and $(0,1)$ respectively. Other choices of $(p, q)$ are possible, but we do not discuss them here. See [25] for more detail.
} 

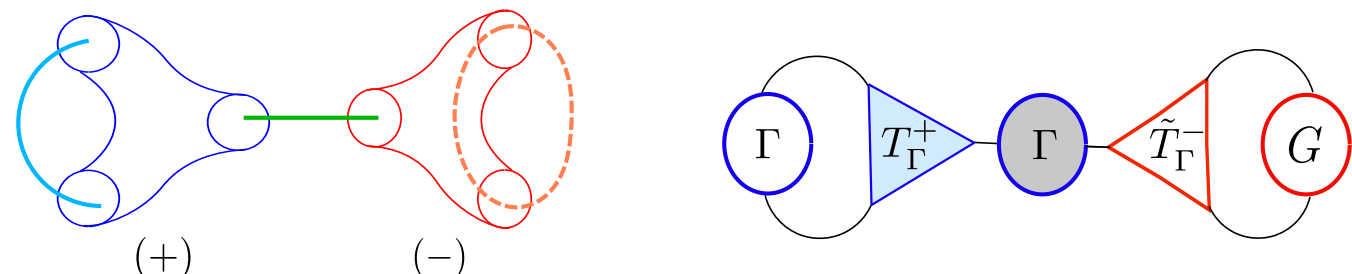

(a) A pair-of-pants decomposition.
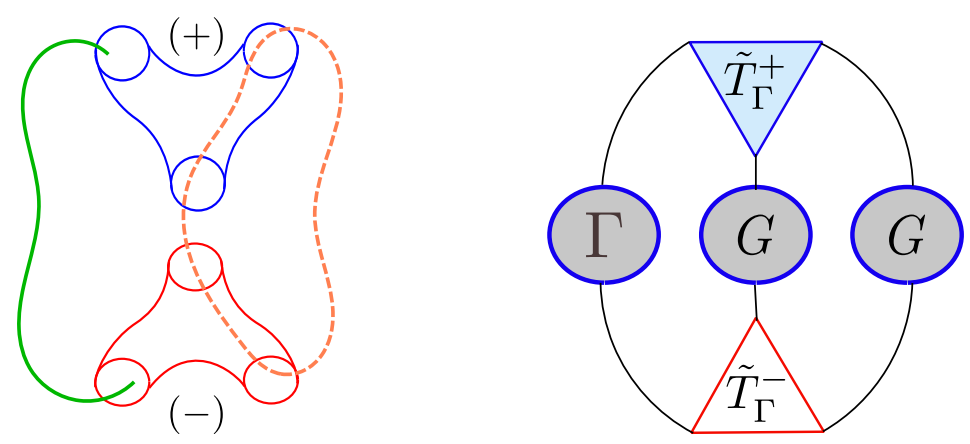

(b) Another pair-of-pants decomposition.

Figure 15. Two ways of decomposing the UV curve $\tilde{\mathcal{C}}_{2,0}$ (labelled as $a$ and $b$ ). The shaded node refers to $\mathcal{N}=1$ gauging whereas unshaded node refers to $\mathcal{N}=2$ gauging.

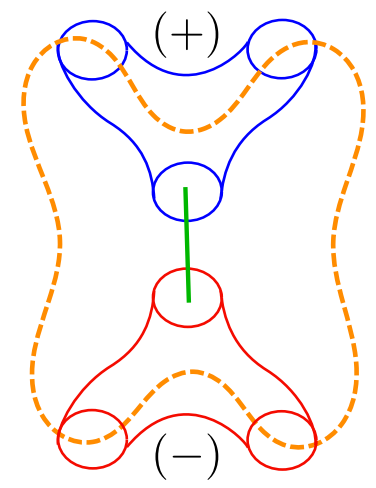

Figure 16. The UV curve $\tilde{\mathcal{C}}_{2,0}^{\prime}$. The twist line (orange) wraps around two holes. The pair-of-pants decomposition reveals that we get the same theory as the case where the twist line wraps only one hole.

yield two dual frames for the same theory. See figure 15. The first one will yield a $T_{\Gamma}$ block and a $\tilde{T}_{\Gamma}$ block with $\Gamma_{\mathcal{N}=2} \times \Gamma_{\mathcal{N}=2} \times G_{\mathcal{N}=1}$ gauge symmetry glued with $\mathcal{N}=1$ and $\mathcal{N}=2$ vector multiplets, while the second decomposition will yield two $\tilde{T}_{\Gamma}$ blocks with $\Gamma_{\mathcal{N}=1} \times G_{\mathcal{N}=1} \times G_{\mathcal{N}=1}$ gauge symmetry with all $\mathcal{N}=1$ vectors.

Just as in the case of $\mathcal{N}=2$, we can consider another UV curve $\tilde{\mathcal{C}}_{2,0}^{\prime}$, where the twist line wraps around two holes of the UV curve. The pair-of-pants decomposition as in figure 16 reveals that it yields same theory as $\tilde{\mathcal{C}}_{2,0}$ even though $\tilde{\mathcal{C}}_{2,0}$ is topologically distinct from $\tilde{\mathcal{C}}_{2,0}^{\prime}$. Therefore, there is only one physically inequivalent configuration for the twist line. 
As we have seen in the case of $\mathcal{N}=2$ theories, we can argue that there is a unique physically inequivalent configuration of the twist line for arbitrary genus $g \geq 2$. The coloring of the pair-of-pants does not have any effect on the proof of our statements about the twist loop, upon replacing S-dualities by IR dualities.

\section{Misleading anomalies and the superconformal indices}

In this section, we compute the 't Hooft anomalies and superconformal indices to verify the dualities. Also, we find that the theory $\mathcal{T}\left[\mathcal{C}_{g, n}\right]$ given by the UV curve without the twist loop and the theory $\mathcal{T}\left[\tilde{\mathcal{C}}_{g, n}\right]$ given by the same curve but with the twist loop share the same anomalies, but have distinct indices.

\subsection{Central charges of the $\mathcal{N}=2$ theories}

For any $4 d \mathcal{N}=2 \mathrm{SCFT}$, the conformal anomalies $(a, c)$ are determined in terms of the 't Hooft anomalies [35]:

$$
\operatorname{Tr} R_{\mathcal{N}=2}=\operatorname{Tr} R_{\mathcal{N}=2}^{3}=2\left(n_{v}-n_{h}\right), \quad \operatorname{Tr} R_{\mathcal{N}=2} I_{3}^{2}=\frac{1}{2} n_{v},
$$

where $I_{3}$ and $R_{\mathcal{N}=2}$ are the Cartans of the R-symmetry $\mathrm{SU}(2)_{R} \times \mathrm{U}(1)_{r}$. It is sometimes more convenient to use the notion of the effective number of hypermultiplets and vector multiplets $n_{h}, n_{v}$ instead of using $a$ and $c$. They are related by

$$
a=\frac{1}{24} n_{h}+\frac{5}{24} n_{v}, \quad c=\frac{1}{12} n_{h}+\frac{1}{6} n_{v} .
$$

If the theory has a flavor symmetry, we can also define the flavor central charge $k$ as

$$
k \delta^{a b}=-2 \operatorname{Tr} R_{\mathcal{N}=2} T^{a} T^{b},
$$

where $T^{a}$ are the generators of the flavor symmetry.

The central charges for the $T_{\Gamma}$ theory can be computed by adding the local contribution from the punctures and the bulk contribution from the sphere [36]. For each of the maximal punctures, we have

$$
n_{h}=\frac{2}{3} h_{\Gamma}^{\vee} d_{\Gamma}, \quad n_{v}=\frac{2}{3} h_{\Gamma}^{\vee} d_{\Gamma}+\frac{1}{2} r_{\Gamma}-\frac{1}{2} d_{\mathfrak{g}},
$$

where $h_{\Gamma}^{\vee}, r_{\Gamma}$ and $d_{\Gamma}$ are the dual Coxeter number, rank and the dimension of $\Gamma$ respectively and $d_{\mathfrak{g}}$ is the dimension of the $\mathfrak{g}$. If the puncture is untwisted, $\mathfrak{g}=\Gamma$ and if it is twisted, it is given by an appropriate non-simply laced group $G$. They are summarized in table 2 . For the bulk of genus $g$, we get $[18,37]$

$$
n_{h}=(g-1)\left(\frac{4}{3} h_{\Gamma}^{\vee} d_{\Gamma}\right), \quad n_{v}=(g-1)\left(\frac{4}{3} h_{\Gamma}^{\vee} d_{\Gamma}+r_{\Gamma}\right) .
$$

Adding up the contributions from the three maximal punctures and the $g=0$ bulk, we get the anomalies for the $T_{\Gamma}$ theory as

$$
n_{v}=\frac{2}{3} h_{\Gamma}^{\vee} d_{\Gamma}+\frac{1}{2} r_{\Gamma}-\frac{3}{2} d_{\Gamma}, \quad n_{h}=\frac{2}{3} h_{\Gamma}^{\vee} d_{\Gamma}, \quad k_{\Gamma}=2 h_{\Gamma}^{\vee},
$$

where $k_{\Gamma}$ is the flavor central charge for each of the three $\Gamma$ symmetries. 


\begin{tabular}{|c|ccccccccc|}
\hline & $A_{n-1}$ & $B_{n}$ & $C_{n}$ & $D_{n}$ & $E_{6}$ & $E_{7}$ & $E_{8}$ & $F_{4}$ & $G_{2}$ \\
\hline$d$ & $n^{2}-1$ & $n(2 n+1)$ & $n(2 n+1)$ & $n(2 n-1)$ & 78 & 129 & 248 & 52 & 14 \\
$h^{\vee}$ & $n$ & $2 n-1$ & $n+1$ & $2 n-2$ & 12 & 18 & 30 & 9 & 4 \\
\hline
\end{tabular}

Table 2. The dimension $d$ and the dual Coxeter number $h^{\vee}$ for all simple Lie groups.

The $\tilde{T}_{\Gamma}$ theory has two twisted punctures and one untwisted puncture, so that

$$
n_{v}=\frac{2}{3} h_{\Gamma}^{\vee} d_{\Gamma}+\frac{1}{2} r_{\Gamma}-d_{G}-\frac{1}{2} d_{\Gamma}, \quad n_{h}=\frac{2}{3} h_{\Gamma}^{\vee} d_{\Gamma}, \quad k_{\Gamma}=2 h_{\Gamma}^{\vee}, \quad k_{G}=2 h_{G}^{\vee},
$$

where $k_{G}$ is the flavor central charge corresponding to the twisted punctures. Notice that the central charge formulae for the $\tilde{T}_{\Gamma}$ are almost identical to that of the $T_{\Gamma}$ theory except for the last term for $n_{v}$. For example, the number of effective vector multiplets $n_{v}$ and hypermultiplets $n_{h}$ for the $T_{\mathrm{SO}(2 N)}$ and $\tilde{T}_{\mathrm{SO}(2 N)}$ theory is given as follows [23]:

$$
\begin{aligned}
& n_{v}\left(T_{\mathrm{SO}(2 N)}\right)=\frac{1}{3} N(N-2)(8 N-5), \\
& n_{v}\left(\tilde{T}_{\mathrm{SO}(2 N)}\right)=\frac{1}{3}(N-1)\left(8 N^{2}-13 N+3\right), \\
& n_{h}\left(T_{\mathrm{SO}(2 N)}\right)=n_{h}\left(\tilde{T}_{\mathrm{SO}(2 N)}\right)=\frac{4}{3} N(N-1)(2 N-1) .
\end{aligned}
$$

Since we know the central charges for the building blocks, one can obtain the central charges for the theories corresponding to the arbitrary UV curve of the higher genus by adding the contribution from each of the pairs-of-pants and the vector multiplets.

Central charges in the presence of the twist line. Let us consider two Riemann surfaces $\mathcal{C}_{g, n}$ and $\tilde{\mathcal{C}}_{g, n}$, each with genus $g$ with $n$ punctures. Let us assume all the punctures are of the untwisted maximal type. The $\tilde{\mathcal{C}}_{g, n}$ has a twist loop with the same geometry as $\mathcal{C}_{g, n}$. See figure 2, for example. Now we perform a pair-of-pants decomposition of $\mathcal{C}_{g, n}$ and $\tilde{\mathcal{C}}_{g, n}$ and write down the theory in term of $T_{\Gamma}$ and $\tilde{T}_{\Gamma}$, where they are glued with the $\mathcal{N}=2$ vector multiplets. (See figure 3.) For the surface $\mathcal{C}_{g, n}$ without twist loop, we have $2 g-2+n$ pairsof-pants, each of them representing $T_{\Gamma}$ block and $3 g-3+n$ of the gauge nodes with group $\Gamma$.

For the $\tilde{\mathcal{C}}_{g, n}$ with a twist loop, some of the $T_{\Gamma}$ blocks are replaced by the $\tilde{T}_{\Gamma}$ block and also some of the gauge groups are replaced by $G$. The number of the $\tilde{T}_{G}$ blocks depends on the choice of the dual frames, but when ever there is a twist line flowing through a pair-of-pants, it should replace the gauge group $\Gamma$ by $G$. Also this is the case since we assume that the twist line is forming a loop. Therefore, the effective number of vector multiplets is given by

$$
n_{v}=(2 g-2+n) n_{v}\left(T_{\Gamma}\right)+(3 g-3+n) d_{\Gamma}+2 \alpha\left[n_{v}\left(\tilde{T}_{\Gamma}\right)-n_{v}\left(T_{\Gamma}\right)+d_{G}-d_{\Gamma}\right],
$$

where $\alpha$ depends on the dual frames. When $\alpha=0$, it describes the case without the twist loop. Now, from (3.6) and (3.7), we have

$$
\left[n_{v}\left(\tilde{T}_{\Gamma}\right)-n_{v}\left(T_{\Gamma}\right)+d_{G}-d_{\Gamma}\right]=0 .
$$


Hence, the presence of the twist line or the choice of the dual frame does not affect the effective number of vector multiplets. Similarly, the effective number of hypermultiplets are given by

$$
n_{h}=(2 g-2+n) n_{h}\left(T_{\Gamma}\right)+2 \alpha\left[n_{h}\left(\tilde{T}_{\Gamma}\right)-n_{h}\left(T_{\Gamma}\right)\right]=(2 g-2+n) n_{h}\left(T_{\Gamma}\right) .
$$

Therefore, a twist loop does not alter the effective number of hypermultiplets as well. To summarize, the central charges $a$ and $c$ are unaltered even if we add a twist loop to the UV curve, namely the theories associated to the UV curves $\mathcal{C}_{g, n}$ and $\tilde{\mathcal{C}}_{g, n}$ have the same anomaly coefficients. Note that all of the flavor central charges match as well, as long as the punctures are of the same type.

The matching of anomalies is rather surprising since the theories corresponding to each curve is not the same! One might think that this is a signal that they are dual to each other, but as we will see later, the superconformal indices for these two theories are different. The two theories having the identical anomaly coefficients have distinct superconformal indices if all the punctures are untwisted, so they are not in the same universality class. We also find that adding a twisted puncture makes the theory dual to each other as we have seen in section 2.2. For this case, the indices match as well.

Coulomb branch operators. Let us also mention that the theories corresponding to $\mathcal{C}_{g, n}$ and $\tilde{\mathcal{C}}_{g, n}$ have the identical Coulomb branch as well. The $T_{\Gamma}$ and the $\tilde{T}_{\Gamma}$ theories have their own Coulomb branches. One can obtain the dimensions of the operators parametrizing the Coulomb branch for the $\Gamma=\mathrm{SO}(2 N)$ using the dualities described in figure 10 or 11 . Both $T_{\mathrm{SO}(2 N)}$ and $\tilde{T}_{\mathrm{SO}(2 N)}$ theories have the Coulomb branch operators $u_{i, j}$ with the scaling dimensions

$$
\begin{aligned}
& {\left[u_{i, j}\right]=2 i+2, \quad i=1, \ldots, N-2, \quad j=1, \ldots, 2 i,} \\
& {\left[u_{N-1, j}\right]=N, \quad\left\{\begin{array}{l}
j=1, \ldots N-2 \text { for } T_{\mathrm{SO}(2 N)} \\
j=1, \ldots N-1 \text { for } \tilde{T}_{\mathrm{SO}(2 N)}
\end{array} .\right.}
\end{aligned}
$$

Therefore the Coulomb operators for the $T_{\mathrm{SO}(2 N)}$ and the $\tilde{T}_{\mathrm{SO}(2 N)}$ are almost identical except for the extra dimension $N$ operator in the $\tilde{T}_{\mathrm{SO}(2 N)}$ theory. Note that once we form a one-punctured torus by gauging the diagonal subgroup of $\operatorname{SO}(2 N)^{2}$ or $\operatorname{USp}(2 N-2)^{2}$ of the $T_{\mathrm{SO}(2 N)}$ or the $\tilde{T}_{\mathrm{SO}(2 N)}$ theory, we get the same set of Coulomb branch operators. One can easily verify that the set of Coulomb branch operators for the higher genus UV curve does not change upon including a loop of twist line. A combination of the conformal anomalies is determined by the dimensions of the Coulomb branch operators as [38]

$$
2 a-c=\frac{1}{4} \sum_{i}\left(2\left[\mathcal{O}_{i}\right]-1\right),
$$

where the sum is over all the Coulomb branch operators. Therefore, this combination of anomalies remains identical upon adding twist line, as we have already shown. 


\begin{tabular}{|c|c|c|c|c|}
\hline & $\operatorname{Tr} J_{+}=\operatorname{Tr} J_{+}^{3}$ & $\operatorname{Tr} J_{-}=\operatorname{Tr} J_{-}^{3}$ & $\operatorname{Tr} J_{+} J_{-}^{2}$ & $\operatorname{Tr} J_{+}^{2} J_{-}$ \\
\hline$T_{\Gamma}^{+}$ & 0 & $r_{\Gamma}-3 d_{\Gamma}$ & 0 & $\frac{4}{3} h_{\Gamma}^{\vee} d_{\Gamma}+r_{\Gamma}-3 d_{\Gamma}$ \\
\hline$\tilde{T}_{\Gamma}^{+}$ & 0 & $r_{\Gamma}-d_{\Gamma}-2 d_{G}$ & 0 & $\frac{4}{3} h_{\Gamma}^{\vee} d_{\Gamma}+r_{\Gamma}-d_{\Gamma}-2 d_{G}$ \\
\hline$T_{\Gamma}^{-}$ & $r_{\Gamma}-3 d_{\Gamma}$ & 0 & $\frac{4}{3} h_{\Gamma}^{\vee} d_{\Gamma}+r_{\Gamma}-3 d_{\Gamma}$ & 0 \\
\hline$\tilde{T}_{\Gamma}^{-}$ & $r_{\Gamma}-d_{\Gamma}-2 d_{G}$ & 0 & $\frac{4}{3} h_{\Gamma}^{\vee} d_{\Gamma}+r_{\Gamma}-d_{\Gamma}-2 d_{G}$ & 0 \\
\hline$\Gamma^{+}$ & 0 & $2 d_{\Gamma}$ & 0 & $2 d_{\Gamma}$ \\
\hline$G^{+}$ & 0 & $2 d_{G}$ & 0 & $2 d_{G}$ \\
\hline$\Gamma^{-}$ & $2 d_{\Gamma}$ & 0 & $2 d_{\Gamma}$ & 0 \\
\hline$G^{-}$ & $2 d_{G}$ & 0 & $2 d_{G}$ & 0 \\
\hline$\Gamma$ & $d_{\Gamma}$ & $d_{\Gamma}$ & $d_{\Gamma}$ & $d_{\Gamma}$ \\
\hline$G$ & $d_{G}$ & $d_{G}$ & $d_{G}$ & $d_{G}$ \\
\hline
\end{tabular}

Table 3. Anomaly coefficients for the building blocks of the $\mathcal{N}=1$ class $\mathcal{S}$ theory. $\Gamma$ and $G$ denotes the $\mathcal{N}=1$ gauge nodes, and $\Gamma^{\sigma}, G^{\sigma}$ denote the $\mathcal{N}=2$ gauge nodes with color $\sigma$.

\subsection{Anomaly coefficients of the $\mathcal{N}=1$ theories}

Now let us generalize our discussion to the $\mathcal{N}=1$ theories. Any class $\mathcal{S}$ theory preserves $\mathrm{U}(1)_{+} \times \mathrm{U}(1)_{-}$global symmetry whose generators we denote as $J_{+}, J_{-}$. Sometimes we use $\mathrm{U}(1)_{R_{0}} \times \mathrm{U}(1)_{\mathcal{F}}$ where $R_{0}=\frac{1}{2}\left(J_{+}+J_{-}\right)$and $\mathcal{F}=\frac{1}{2}\left(J_{+}-J_{-}\right)$. For the $\mathcal{N}=2$ theory, they can be written in terms of $\mathcal{N}=2 R$-charges as

$$
R_{0}=\frac{1}{2} R_{\mathcal{N}=2}+I_{3}, \quad \mathcal{F}=-\frac{1}{2} R_{\mathcal{N}=2}+I_{3}
$$

When constructing an $\mathcal{N}=1$ theory by gluing the $T_{\Gamma}$ or $\tilde{T}_{\Gamma}$ blocks, we assign color $\sigma_{i}$ to each block and one linear combination of $\mathrm{U}(1)_{\mathcal{F}}$ for the blocks

$$
\mathcal{F}=\sum_{i} \sigma_{i} \mathcal{F}_{i}
$$

is preserved upon gluing. Here $\mathcal{F}_{i}$ is the $\mathrm{U}(1)$ symmetry acting only on the $i$-th block. Let us write $T_{\Gamma}^{\sigma}$ and $\tilde{T}_{\Gamma}^{\sigma}$ to denote the coloring for each building block. Now, using the equation (3.1), the anomaly coefficients can be computed. The results are in table 3.

Let us mention that the anomalies for the class $\mathcal{S}$ theories having neither punctures nor twist lines can be obtained from integrating the anomaly polynomial for the $6 \mathrm{~d} \mathcal{N}=(2,0)$ theory [39-41] as done in $[18,19,37]$.

Anomaly coefficients in the presence of the twist line. Suppose we have a UV curve $\mathcal{C}_{g, n}$ with the normal bundles, having degrees $(p, q)$. Let us assume that all the punctures are of the maximal type. Now, consider a colored pair-of-pants decomposition such that the number of $\mathcal{N}=2$ gluing with + color is $x$, the number of $\mathcal{N}=2$ gluing with - color 
is $y$ and the number of $\mathcal{N}=1$ gluing is $z$. This leads to the following anomaly coefficients:

$$
\begin{aligned}
\operatorname{Tr} J_{+} & =\operatorname{Tr} J_{+}^{3}=q\left(r_{\Gamma}-3 d_{\Gamma}\right)+(2 y+z) d_{\Gamma}, \\
\operatorname{Tr} J_{-} & =\operatorname{Tr} J_{-}^{3}=p\left(r_{\Gamma}-3 d_{\Gamma}\right)+(2 x+z) d_{\Gamma}, \\
\operatorname{Tr} J_{+} J_{-}^{2} & =2 q\left(\frac{2}{3} h_{\Gamma}^{\vee} d_{\Gamma}+\frac{1}{2} r_{\Gamma}-\frac{3}{2} d_{\Gamma}\right)+(2 x+z) d_{\Gamma}, \\
\operatorname{Tr} J_{+}^{2} J_{-} & =2 p\left(\frac{2}{3} h_{\Gamma}^{\vee} d_{\Gamma}+\frac{1}{2} r_{\Gamma}-\frac{3}{2} d_{\Gamma}\right)+(2 x+z) d_{\Gamma} .
\end{aligned}
$$

Now we use following identities: ${ }^{7}$

$$
(2 x+z)+(2 y+z)=3(p+q), \quad(2 x+z)-(2 y+z)=3(p-q) .
$$

to recast the anomaly coefficients as:

$$
\begin{aligned}
\operatorname{Tr} J_{+} & =\operatorname{Tr} J_{+}^{3}=q r_{\Gamma}, \\
\operatorname{Tr} J_{-} & =\operatorname{Tr} J_{-}^{3}=p r_{\Gamma}, \\
\operatorname{Tr} J_{+} J_{-}^{2} & =2 q\left(\frac{2}{3} h_{\Gamma}^{\vee} d_{\Gamma}+\frac{1}{2} r_{\Gamma}\right), \\
\operatorname{Tr} J_{+}^{2} J_{-} & =2 p\left(\frac{2}{3} h_{\Gamma}^{\vee} d_{\Gamma}+\frac{1}{2} r_{\Gamma}\right) .
\end{aligned}
$$

Now, let us introduce a twist loop passing through a cycle on the UV curve. Upon colored pair-of-pants decomposition, it will pass through a number of + type and - type pants. Let us write the sequence of the color of the pants as $\sigma_{1} \sigma_{2} \cdots \sigma_{\ell}$. The sequence is cyclic so that $\sigma_{\ell+1}=\sigma_{1}$. As the twist line passes through the pairs-of-pants, it replaces $T_{\Gamma}^{\sigma_{i}}$ by $\tilde{T}_{\Gamma}^{\sigma_{i}}$ and also changes the gauge group $\Gamma^{\sigma_{i} \sigma_{i+1}}$ between the pants $i$ and $j$ by $G^{\sigma_{i} \sigma_{i+1}}$. Here +- and -+ refer to $\mathcal{N}=1$ vector multiplet, whereas ++ and -- refer to $\mathcal{N}=2$ vector. Now, the effect of the twist line to the anomalies can schematically be written as

$$
\begin{aligned}
\delta_{\mathcal{A}} & =\sum_{i=1}^{\ell}\left(\tilde{T}_{\Gamma}^{\sigma_{i}}-T_{\Gamma}^{\sigma_{i}}+G^{\sigma_{i} \sigma_{i+1}}-\Gamma^{\sigma_{i} \sigma_{i+1}}\right) \\
& =\sum_{i=1}^{\ell}\left[\frac{1}{2}\left(\tilde{T}_{\Gamma}^{\sigma_{i}}-T_{\Gamma}^{\sigma_{i}}\right)+\frac{1}{2}\left(\tilde{T}_{\Gamma}^{\sigma_{i+1}}-T_{\Gamma}^{\sigma_{i+1}}\right)+\left(G^{\sigma_{i} \sigma_{i+1}}-\Gamma^{\sigma_{i} \sigma_{i+1}}\right)\right]
\end{aligned}
$$

where each symbol means the anomaly coefficients of the corresponding block. It is easy to verify that the term in the square bracket vanishes. Therefore adding a twist loop does not alter the anomaly coefficients.

\footnotetext{
${ }^{7}$ If a gluing is done between two pairs-of-pants labelled by $\sigma_{i}$ and $\sigma_{j}$, let us assign the value $F_{\langle i j\rangle}=\sigma_{i}+\sigma_{j}$ to the gluing, denoted by $\langle i j\rangle$. Now we will consider the sum $I=\sum_{i j}\left(\sigma_{i}+\sigma_{j}\right)$ in two different ways. Since each pair-of-pants has 3 punctures, $I=3(p-q)$. On the other hand, $I=\sum_{\text {gluing }} F_{\langle i j\rangle}$, now $F_{\langle i j\rangle}$ is 0 if we have $\mathcal{N}=1$ gluing, $F_{\langle i j\rangle}= \pm 2$ respectively for gluing two \pm type pairs-of-pants. Hence, $I=2 x-2 y+0$ and $I=2(x-y)$. So we have $2(x-y)=3(p-q)$. Note that if we do not weight the gluing by their signs, then we would have obtained $2(x+y+z)=3(p+q)$.
} 
Anomaly coefficients in the presence of punctures. Let us derive the formula for the anomaly coefficients in the presence of $n_{\Gamma \pm} / n_{G \pm}$ number of $\Gamma / G$-type punctures (untwisted/twisted full punctures) with color \pm . We define $n_{ \pm}=n_{G \pm}+n_{\Gamma \pm}$. While $n_{G \pm}$ is an even number, $n_{\Gamma \pm}$ can be even or odd. Our argument about the twist loop still holds in the presence of the puncture, so the result is not affected by the twist.

When $n_{\Gamma \pm}$ are all even, we can glue all the punctures pairwise with $\mathcal{N}=2$ vector multiplets in some particular dual frame. This yields the UV curve without a puncture, with the degrees of the line bundles given by $\left(p+n_{+}, q+n_{-}\right)$. Now we can calculate the anomalies corresponding to this modified surface and then subtract the contribution from the vector multiplets due to the gluing. In this way, we obtain

$$
\begin{aligned}
\operatorname{Tr} J_{+} & =\operatorname{Tr} J_{+}^{3}=\left(q+n_{-}\right) r_{\Gamma}-2 n_{\Gamma-} d_{\Gamma}-2 n_{G-} d_{G}, \\
\operatorname{Tr} J_{-} & =\operatorname{Tr} J_{-}^{3}=\left(p+n_{+}\right) r_{\Gamma}-2 n_{\Gamma+} d_{\Gamma}-2 n_{G+} d_{G}, \\
\operatorname{Tr} J_{+}^{2} J_{-} & =2\left(p+n_{+}\right)\left(\frac{2}{3} h_{\Gamma}^{\vee} d_{\Gamma}+\frac{1}{2} r_{\Gamma}\right)-2 n_{\Gamma+} d_{\Gamma}-2 n_{G+} d_{G}, \\
\operatorname{Tr} J_{+} J_{-}^{2} & =2\left(q+n_{-}\right)\left(\frac{2}{3} h_{\Gamma}^{\vee} d_{\Gamma}+\frac{1}{2} r_{\Gamma}\right)-2 n_{\Gamma-} d_{\Gamma}-2 n_{G-} d_{G} .
\end{aligned}
$$

If any or both of $n_{\Gamma \pm}$ is odd, then we can consider $n_{\Gamma \pm}^{\prime} \equiv n_{\Gamma \pm}-1$ punctures, for whichever one ( + or - or both) is odd. Since $n_{\Gamma \pm}^{\prime}$ is an even number, we can employ the previous trick to compute the anomaly coefficients, which we obtain by substituting $n_{ \pm} \rightarrow n_{ \pm}^{\prime} \equiv n_{ \pm}-1$. In order to get the correct anomaly coefficient, all we have to do is to add the extra pair-of-pants with appropriate label to the rest of the diagram. This is effectively the same as adding a pair-of-pants to the diagram with an $\mathcal{N}=2$ vector multiplet with both of them having the same color assignment as the puncture. Hence, the formula given above (3.21) holds true for this case as well. The diagrams given in figure 17 will elucidate that either we get explicitly an extra $\mathcal{N}=2$ gauge node of the same color as the puncture or we get two extra $\mathcal{N}=1$ vector multiplet while losing one $\mathcal{N}=2$ vector multiplet of opposite color of puncture. It can be easily verified that adding two extra $\mathcal{N}=1$ vector multiplets while losing one $\mathcal{N}=2$ vector multiplet of opposite sign of the puncture is effectively the same as adding one $\mathcal{N}=2$ vector multiplet of the same color as the puncture. All of these different gluing represent dual frames of the same theory.

The punctures also introduce flavor symmetries, hence we have more anomaly coefficients. For a maximal puncture carrying the flavor symmetry group $\mathfrak{g}$ and labelled by the color $\sigma$ we have

$$
\operatorname{Tr} J_{ \pm} T^{a} T^{b}=-\frac{1 \mp \sigma}{2} h_{\mathfrak{g}}^{\vee} \delta^{a b} .
$$

Here $\mathfrak{g}=\Gamma$ if the puncture is untwisted and $\mathfrak{g}=G$ if the puncture is twisted.

\section{$3.3 \quad a$-maximization}

The superconformal $R$ symmetry in infrared is given by:

$$
R_{\mathrm{IR}}=R_{0}+\epsilon \mathcal{F}
$$




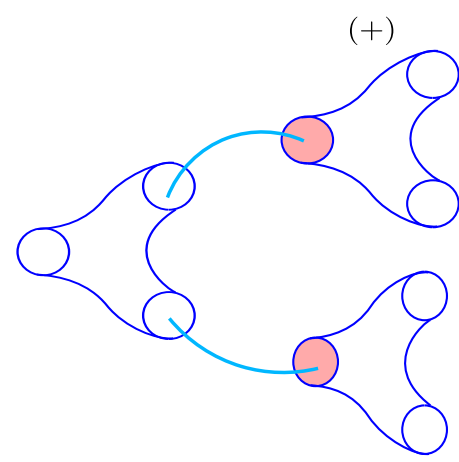

$(+)$

$(+)$

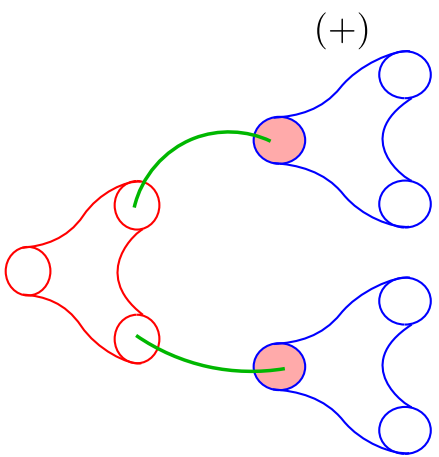

$(-)$

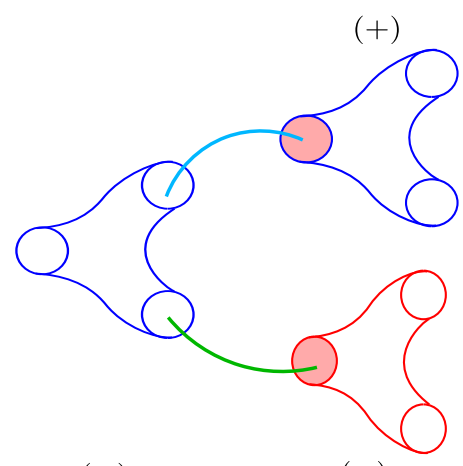

$(+)$

$(-)$

Figure 17. Inclusion of an extra pair-of-pants (left most one in each figure) to the UV curve when $n_{\Gamma \pm}$ is odd. The solid lines denote extra gluing. Green lines denote $\mathcal{N}=1$ gluing while the blue lines are for the $\mathcal{N}=2$ gluing. There are 3 more diagrams we can have, which are obtained by swapping the colors of pairs-of-pants from blue to red and vice-versa in each of the diagrams.

where $\epsilon$, hence $R_{\mathrm{IR}}$ is determined by $a$-maximization [34]. The $a$-function is given by the trace anomalies as

$$
a=\frac{3}{32}\left(3 \operatorname{Tr} R_{\mathrm{IR}}^{3}-\operatorname{Tr} R_{\mathrm{IR}}\right) .
$$

We determine the value of $\epsilon$ by using the trial $R$-charge above and then solving

$$
\left.\frac{\partial a}{\partial \epsilon}\right|_{\epsilon}=0,\left.\quad \frac{\partial^{2} a}{\partial \epsilon^{2}}\right|_{\epsilon}<0 .
$$

If two theories have identical anomaly coefficients, it would imply that the polynomial $a(\epsilon)$ is the same, subsequently, we have the same $\epsilon$ as a maximizing solution. The only thing that we are required to check is whether there is any accidental symmetry appearing from the decoupling of any operators since the operator content of the two theories of interest might be different. Should we have any operator violating the unitarity bound, that gets decoupled, and we are required to do the $a$-maximization once again upon removing the contributions coming from those operators violating the unitary bound [42].

In our case, as long as $p>0, q>0$, it is sufficient to verify whether $|\epsilon| \leq \frac{1}{3}$, since the chiral operators of lowest $R$-charge have $\left(J_{+}, J_{-}\right)=(2,0)$ or $(0,2)$. We find that for a UV curve $\mathcal{C}_{g, 0}$ with the degrees of the line bundles given by $p$ and $q$, the trial $a$-function comes out to be:

$$
a(\epsilon)=-\frac{9}{32} d_{\Gamma} h_{\Gamma}^{\vee} x \epsilon^{2}+\frac{9 d_{\Gamma} h_{\Gamma}^{\vee} x}{32}-\frac{9 d_{\Gamma} h_{\Gamma}^{\vee} y}{32} \epsilon^{3}+\frac{9 d_{\Gamma} h_{\Gamma}^{\vee} y}{32} \epsilon+\frac{3 r_{\Gamma} x}{16}-\frac{9 r_{\Gamma} y}{32} \epsilon^{3}+\frac{3 r_{\Gamma} y \epsilon}{32}
$$

where $x=\frac{p+q}{2}$ and $y=\frac{p-q}{2}$. This is true irrespective of presence/absence of twist line loops on the UV curve. Here $d_{\Gamma}$ is the dimension of flavor symmetry group $\Gamma$ associated with untwisted puncture and $h_{\Gamma}^{\vee}$ is the dual Coxeter number of $\Gamma$. We define $g_{\Gamma}=\frac{r_{\Gamma}}{d_{\Gamma} h_{\Gamma}^{\nabla}}$ where $r_{\Gamma}$ is the rank of the group $\Gamma$ and $t=\frac{p-q}{p+q}$. The $\epsilon$ that maximizes the trial $a$-function is given by

$$
\epsilon(t)=\frac{\sqrt{\left(g_{\Gamma}+1\right)\left(g_{\Gamma}+3\right) t^{2}+1}-1}{3\left(g_{\Gamma}+1\right) t}
$$



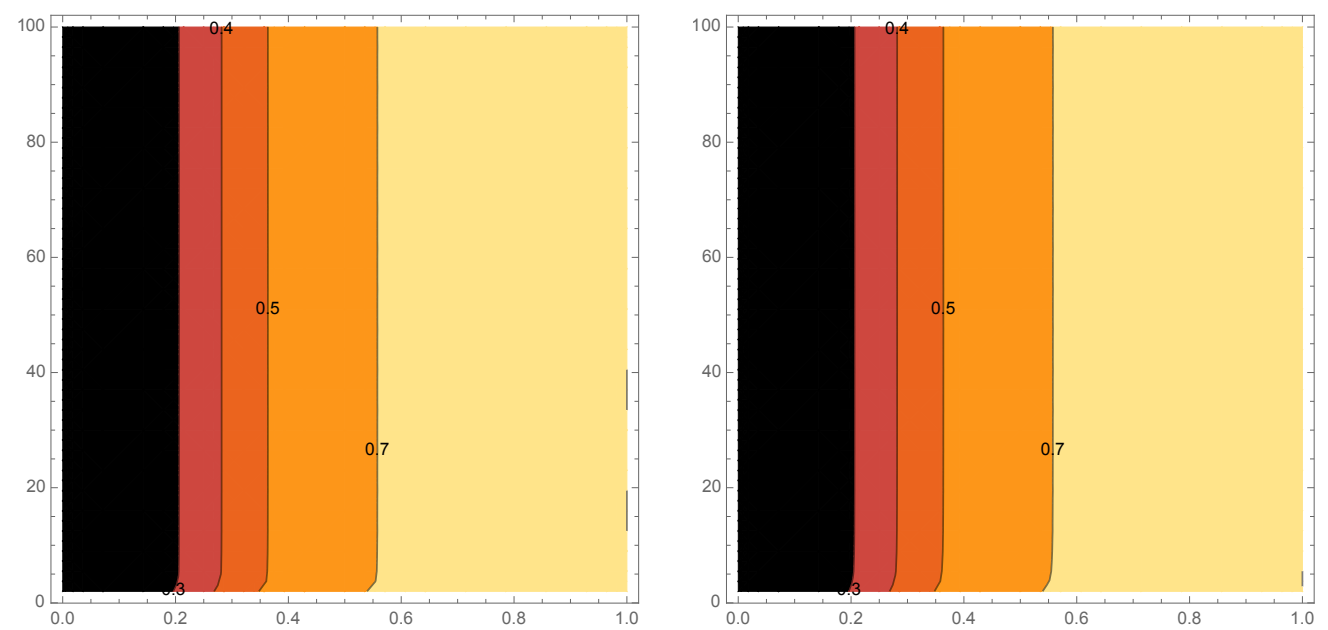

Figure 18. A contour plot of $3 \epsilon$ that maximizes $a(\epsilon)$ for $\Gamma=D_{n}$ and $\Gamma=A_{n-1}$. The $Y$ axis denotes $n$, and the $X$ axis is for the $t=\frac{p-q}{p+q}$, parametrizing the choice of the normal bundles. Note the $t$ has to be in this range $|t| \leq 1$. Since, $\epsilon(-t)=-\epsilon(t)$, it is sufficient to plot for $0 \leq t \leq 1$.

For $A_{n-1}$, we have $\frac{1}{g_{\Gamma}}=n(n+1)$, for $D_{n}$ we have $\frac{1}{g_{\Gamma}}=(2 n-1)(2 n-2)$; for $E_{6}$, we have $\frac{1}{g_{\Gamma}}=156$. For each of these cases, we find $|\epsilon| \leq \frac{1}{3}$ as long as $p>0, q>0$. See figure 18 for the case of $\Gamma=D_{n}$ and $A_{n-1}$. Therefore none of the operators decouples along the renormalization group flow as long as $p>0, q>0$ and UV curve does not have any puncture.

\subsection{Superconformal index}

In this section, we discuss the superconformal indices of the class $\mathcal{S}$ theories that we studied so far. The superconformal index is a supersymmetric partition function that captures the multiplicities of the short multiplets in a SCFT. It provides a strong check of the supersymmetric dualities. We remark that most of the discussions in the current subsection are already known. We would simply like to highlight the effect of the twist lines in the computation of the index. We refer to the original papers [26-29, 32] and the review article [43] and the references therein for more details. We would like to focus on the effect of the outer-automorphism twist in the index, which has been discussed in [16, 23, 30,31].

The superconformal index for an $\mathcal{N}=2$ class $\mathcal{S}$ theory can be written as a partition function of certain $2 \mathrm{~d}$ topological field theory (TQFT) on the UV curve $\mathcal{C}_{g, n}$ of genus $g$ and $n$ punctures (here we assume all the punctures to be maximal) as

$$
\mathcal{I}_{\mathcal{C}_{g, n}}(p, q, t ; \boldsymbol{a})=\sum_{\boldsymbol{\lambda} \in R_{\Gamma}} C_{\boldsymbol{\lambda}}(p, q, t)^{2 g-2+n} \prod_{I=1}^{n} \psi_{\boldsymbol{\lambda}}^{(I)}\left(p, q, t ; \boldsymbol{a}_{I}\right),
$$

where $C_{\boldsymbol{\lambda}}$ is called the structure constant of the TQFT and $\psi_{\boldsymbol{\lambda}}^{(I)}$ are the wave functions associated to the punctures. The parameters $p, q, t$ are the fugacities associated to some linear combinations of the Cartans of the superconformal group $\mathrm{SU}(2,2 \mid 2)$, and $\boldsymbol{a}_{I}$ are the fugacities associated to the global symmetry coming from each puncture. Notice that the 
sum is over all the representations $R_{\Gamma}$ of the group $\Gamma$ labeling the class $\mathcal{S}$ theories. So far, we have assumed that there is no twist lines on the UV curve.

For $g=0, n=3$, we obtain the index for the $T_{\Gamma}$ theory given as

$$
\mathcal{I}_{T_{\Gamma}}\left(\boldsymbol{a}_{1}, \boldsymbol{a}_{2}, \boldsymbol{a}_{3}\right)=\sum_{\boldsymbol{\lambda} \in R_{\Gamma}} C_{\boldsymbol{\lambda}} \psi_{\boldsymbol{\lambda}}\left(\boldsymbol{a}_{1}\right) \psi_{\boldsymbol{\lambda}}\left(\boldsymbol{a}_{2}\right) \psi_{\boldsymbol{\lambda}}\left(\boldsymbol{a}_{3}\right)
$$

where we omitted the dependence on the fugacities $p, q, t$. The wave functions satisfy

$$
\oint[d \boldsymbol{z}]_{\Gamma} I_{\mathrm{vec}, \Gamma}(\boldsymbol{z}) \psi_{\boldsymbol{\lambda}}(\boldsymbol{z}) \psi_{\boldsymbol{\mu}}(\boldsymbol{z})=\delta_{\boldsymbol{\lambda} \boldsymbol{\mu}}
$$

where $I_{\mathrm{vec}, \Gamma}$ is the index for the vector multiplets and $[d \boldsymbol{z}]_{\Gamma}$ is the Haar measure for the gauge group $\Gamma$. From this orthonormality condition, one can easily obtain the index formula (3.28) for the arbitrary UV curve $\mathcal{C}_{g, n}$ by multiplying the contributions from each block and the vector multiplet and then by integrating over the gauge groups. The formula (3.28) is independent of the pair-of-pants decomposition, which is consistent with the duality.

When there is a twist line, we have $\tilde{T}_{\Gamma}$ blocks along with the $T_{\Gamma}$ theories. The index for the $\tilde{T}_{\Gamma}$ is given by

$$
\mathcal{I}_{T_{G}}\left(\boldsymbol{a}, \boldsymbol{b}_{1}, \boldsymbol{b}_{2}\right)=\sum_{\boldsymbol{\lambda} \in R_{G}} C_{\boldsymbol{\lambda}} \psi_{\boldsymbol{\lambda}}(\boldsymbol{a}) \tilde{\psi}_{\boldsymbol{\lambda}}\left(\boldsymbol{b}_{1}\right) \tilde{\psi}_{\boldsymbol{\lambda}}\left(\boldsymbol{b}_{2}\right)
$$

where the wave functions are changed appropriately to that of the twisted punctures. Here the summation is over the representations $R_{G}$ of the group $G$, instead of $\Gamma$. This means that for the untwisted punctures $\psi_{\boldsymbol{\lambda}}$, we sum over only over the representations of $\Gamma$ that are invariant under the $\mathbb{Z}_{2}$ outer-automorphism action. The wave functions for the twisted punctures are orthonormal under the vector multiplet measure for the gauge group $G$ :

$$
\oint[d \boldsymbol{z}]_{G} I_{\mathrm{vec}, G}(\boldsymbol{z}) \tilde{\psi}_{\boldsymbol{\lambda}}(\boldsymbol{z}) \tilde{\psi}_{\boldsymbol{\mu}}(\boldsymbol{z})=\delta_{\boldsymbol{\lambda} \boldsymbol{\mu}} .
$$

Now, one can easily obtain the index in the presence of a twist line as

$$
\mathcal{I}_{\tilde{\mathcal{C}}_{g, n}}(p, q, t ; \boldsymbol{a})=\sum_{\boldsymbol{\lambda} \in R_{G}} C_{\boldsymbol{\lambda}}(p, q, t)^{2 g-2+n} \prod_{I=1}^{n} \psi_{\boldsymbol{\lambda}}^{(I)}\left(p, q, t ; \boldsymbol{a}_{I}\right),
$$

when all the external punctures are untwisted. Notice that the only difference between (3.28) and (3.33) is the domain of summation. This clearly implies that the indices for the theory with the twist line differ from the one without the twist line. Also, there is no particular choice of the twist loop either. This is consistent our claim that there is only one physically inequivalent configuration of the twist loop.

Let us consider some simple examples to see this explicitly. Consider the Schur limit of the index, which takes $p \rightarrow 0, q=t$. Then the wave function and the structure constant can be written as

$$
\psi_{\boldsymbol{\lambda}}(\boldsymbol{a})=\mathrm{PE}\left[\frac{q}{1-q} \chi_{\mathrm{adj}}(\boldsymbol{a})\right] \chi_{\boldsymbol{\lambda}}(a), \quad C_{\boldsymbol{\lambda}}=\prod_{i=1}^{r_{\Gamma}}\left(q^{d_{i}} ; q\right) / \chi_{\boldsymbol{\lambda}}\left(q^{\boldsymbol{\rho}}\right),
$$


where PE denotes the plethystic exponential, and the $q$-Pochammer symbol is given by $(x ; q) \equiv \prod_{n \geq 0}\left(1-x q^{n}\right)$, and $d_{i}$ are the degrees of the Casimirs of $\Gamma$ and $r_{\Gamma}$ is the rank of $\Gamma . \chi_{\boldsymbol{\lambda}}(\boldsymbol{z})$ is the character of the representation $\boldsymbol{\lambda}$ and $\boldsymbol{\rho}$ denotes the Weyl vector of $\Gamma$ and $q^{\rho} \equiv\left(q^{\rho_{1}}, q^{\rho_{2}}, \ldots, q^{\rho_{r_{\Gamma}}}\right)$.

For example, when $g=2, n=0$ and $\Gamma=D_{4}$, we obtain

$$
\mathcal{I}_{\mathcal{C}_{2,0}}(q)=1-2 q^{2}-2 q^{3}-5 q^{4}-2 q^{5}+8 q^{6}+8 q^{7}+24 q^{8}+O\left(q^{9}\right),
$$

for the case without the twist loop. When we have a twist loop, the sum is over the representations of $G=C_{3}$. We get

$$
\mathcal{I}_{\tilde{\mathcal{C}}_{2,0}}(q)=1-2 q^{2}-2 q^{3}-5 q^{4}-2 q^{5}+6 q^{6}+12 q^{7}+26 q^{8}+O\left(q^{9}\right) .
$$

The indices for the two theories indeed differ, and they start to deviate at order 6 .

Let us consider another example: $g=1, n=1$ and $\Gamma=D_{4}$. For this case, we get

$$
\begin{aligned}
\mathcal{I}_{\mathcal{C}_{1,1}(q ; \boldsymbol{a})=}+ & +q \chi_{[0100]}+q^{2}\left(\chi_{[0100]}+\chi_{[2000]}+\chi_{[0020]}+\chi_{[0002]}+\chi_{[0200]}\right) \\
+ & \left(\chi_{[0300]}+\chi_{[2100]}+\chi_{[0102]}+\chi_{[0120]}+\chi_{[0200]}+2 \chi_{[1011]}+\chi_{[0020]}\right. \\
& \left.+\chi_{[2000]}+\chi_{[0002]}+4 \chi_{[0100]}+\chi_{[1000]}+\chi_{[0010]}+\chi_{[0001]}\right) q^{3}+O\left(q^{4}\right) \\
=1 & +28 q+433 q^{2}+4867 q^{3}+44234 q^{4}+343744 q^{5}+O\left(q^{6}\right)
\end{aligned}
$$

for the case without the twist loop. Here $\chi_{R}$ denotes the character of the $\mathrm{SO}(8)$ for the representation given by the Dynkin label $R$. The final line is the result after taking the flavor fugacities $\boldsymbol{a}$ to 1 . On the other hand, when the UV curve has a twist loop, we get

$$
\begin{aligned}
\mathcal{I}_{\tilde{\mathcal{C}}_{1,1}}(q ; \boldsymbol{a})=1 & +q \chi_{[0100]}+q^{2}\left(\chi_{[0100]}+\chi_{[2000]}+\chi_{[0020]}+\chi_{[0002]}+\chi_{[0200]}\right) \\
+ & \left(\chi_{[0300]}+\chi_{[2100]}+\chi_{[0102]}+\chi_{[0120]}+\chi_{[0200]}+2 \chi_{[1011]}+\chi_{[0020]}\right. \\
& \left.+\chi_{[2000]}+\chi_{[0002]}+4 \chi_{[0100]}+\chi_{[1000]}\right) q^{3}+O\left(q^{4}\right) \\
=1 & +28 q+433 q^{2}+4851 q^{3}+43802 q^{4}+337264 q^{5}+O\left(q^{6}\right) .
\end{aligned}
$$

The difference begins at order $q^{3}$. The linear term in the index comes from the conserved current multiplet. Therefore, the global symmetries for both theories are identical and there is no symmetry enhancement from $\mathrm{SO}(8)$ which comes from the puncture.

Let us comment that our discussion can easily be generalized to $\mathcal{N}=1$ class $\mathcal{S}$ theories. Especially, the $\mathcal{N}=1$ index also admits the Schur limit, which gives the identical partition function as the $\mathcal{N}=2$ counterpart. In this limit, the dependence on the degrees of the normal bundles vanishes.

Therefore we see that the theories given by the UV curve $\mathcal{C}_{g, n}$ and the identical curve with the twist loop $\tilde{\mathcal{C}}_{g, n}$ have different superconformal indices, but the same anomalies. Also, we notice that whenever the twisted puncture is introduced, it changes the range of summation from the representations of $\Gamma$ to $G$. Therefore the index does not get altered further upon inserting a new loop of twist line. This is consistent with the duality as stated in (1.1). 


\section{Conclusion}

In this paper, we have studied four-dimensional superconformal theories in class $\mathcal{S}$, in the presence of the outer-automorphism twist line forming a loop on the UV curve. We have found new dualities in the presence of the twist loop and performed checks using anomalies and superconformal index. Quite interestingly, we have found that the theory with a loop of twist line on the UV curve and the theory without the twist line have the identical anomalies, but the indices differ. This provides a simple way of constructing a pair of distinct theories giving identical 't Hooft anomalies.

The examples of misleading anomaly matchings we find are somewhat analogous to the orbifolds in 2d CFTs. As is well-known, orbifolding does not change the central charges of the theory but it does change the theory in a non-trivial way. Our example can be thought of as a higher-dimensional analog of orbifolding. When we obtain the 4d SCFT, we truncate certain degrees of freedom that are not invariant under the outer-automorphism twist. We also get some extra states similar to the twisted sector. But this operation does not change the overall behavior regarding the growth of the number of states. We expect that it is straight-forward to generalize our observations to the theories having some higher dimensional origin. For example, one can consider $4 \mathrm{~d} \mathcal{N}=1$ or $2 \mathrm{~d} \mathcal{N}=(0,2)$ theories coming from $6 \mathrm{~d} \mathcal{N}=(1,0)$ theories on a Riemann surface [44-50] with a twist loop.

In this paper, we have found pairs of theories having the identical anomalies. One interesting question to ask is whether it is possible to have many more theories with identical anomalies. In our examples, the theory has exactly marginal deformations which do not change the anomalies, so technically speaking there are actually infinitely many theories giving the same anomalies. But once we mod out by such exactly marginal deformations, how many distinct theories are there for given anomalies? We were able to distinguish them using the superconformal indices. Can there be another theory giving a different index function with the same anomalies? The lens space index provides even more refined quantity, which can distinguish the theories having different sets of non-local operators [51, 52]. How many theories (up to exactly marginal deformations) with distinct lens space indices exist for a given anomalies? It would be interesting to answer these questions.

Finally, we would like to point out that all the examples of misleading anomaly matching we studied are 'non-Lagrangian' with no known Lagrangian description. It would be interesting to find more examples of conventional Lagrangian gauge theory or find if there is any obstruction to such cases.

\section{Acknowledgments}

We would like to thank Ken Intriligator, Emily Nardoni and Yuji Tachikawa for discussions and correspondence. This work is supported by the US Department of Energy under UCSD's contract de-sc0009919. The work of JS is also supported in part by Hwa-Ahm foundation. 
Open Access. This article is distributed under the terms of the Creative Commons Attribution License (CC-BY 4.0), which permits any use, distribution and reproduction in any medium, provided the original author(s) and source are credited.

\section{References}

[1] N. Seiberg, Electric-magnetic duality in supersymmetric nonAbelian gauge theories, Nucl. Phys. B 435 (1995) 129 [hep-th/9411149] [INSPIRE].

[2] J.H. Brodie, P.L. Cho and K.A. Intriligator, Misleading anomaly matchings?, Phys. Lett. B 429 (1998) 319 [hep-th/9802092] [INSPIRE].

[3] G.S. Vartanov, On the ISS model of dynamical SUSY breaking, Phys. Lett. B 696 (2011) 288 [arXiv: 1009.2153] [INSPIRE].

[4] V. Pestun et al., Localization techniques in quantum field theories, arXiv:1608.02952 [INSPIRE].

[5] D. Gaiotto, $N=2$ dualities, JHEP 08 (2012) 034 [arXiv:0904.2715] [INSPIRE].

[6] D. Gaiotto, G.W. Moore and A. Neitzke, Wall-crossing, Hitchin Systems and the WKB Approximation, arXiv:0907.3987 [INSPIRE].

[7] Y. Tachikawa, Six-dimensional $D_{N}$ theory and four-dimensional SO-USp quivers, JHEP 07 (2009) 067 [arXiv:0905.4074] [INSPIRE].

[8] Y. Tachikawa, $N=2$ S-duality via Outer-automorphism Twists, J. Phys. A 44 (2011) 182001 [arXiv: 1009.0339] [INSPIRE].

[9] O. Chacaltana and J. Distler, Tinkertoys for Gaiotto Duality, JHEP 11 (2010) 099 [arXiv: 1008.5203] [INSPIRE].

[10] O. Chacaltana and J. Distler, Tinkertoys for the $D_{N}$ series, JHEP 02 (2013) 110 [arXiv:1106.5410] [INSPIRE].

[11] T. Nishinaka, The gravity duals of SO/USp superconformal quivers, JHEP 07 (2012) 080 [arXiv:1202.6613] [INSPIRE].

[12] O. Chacaltana, J. Distler and Y. Tachikawa, Gaiotto duality for the twisted $A_{2 N-1}$ series, JHEP 05 (2015) 075 [arXiv: 1212.3952] [INSPIRE].

[13] O. Chacaltana, J. Distler and A. Trimm, Tinkertoys for the Twisted D-Series, arXiv: 1309.2299 [INSPIRE].

[14] O. Chacaltana, J. Distler and A. Trimm, Tinkertoys for the $E_{6}$ theory, JHEP 09 (2015) 007 [arXiv: 1403.4604] [INSPIRE].

[15] O. Chacaltana, J. Distler and A. Trimm, A Family of $4 D \mathcal{N}=2$ Interacting SCFTs from the Twisted $A_{2 N}$ Series, arXiv:1412.8129 [INSPIRE].

[16] O. Chacaltana, J. Distler and A. Trimm, Tinkertoys for the Twisted $E_{6}$ Theory, JHEP 04 (2015) 173 [arXiv: 1501.00357] [INSPIRE].

[17] O. Chacaltana, J. Distler and A. Trimm, Tinkertoys for the Z3-twisted D4 Theory, arXiv: 1601.02077 [INSPIRE].

[18] F. Benini, Y. Tachikawa and B. Wecht, Sicilian gauge theories and $N=1$ dualities, JHEP 01 (2010) 088 [arXiv:0909.1327] [INSPIRE]. 
[19] I. Bah, C. Beem, N. Bobev and B. Wecht, Four-Dimensional SCFTs from M5-Branes, JHEP 06 (2012) 005 [arXiv: 1203.0303] [INSPIRE].

[20] A. Gadde, K. Maruyoshi, Y. Tachikawa and W. Yan, New N=1 Dualities, JHEP 06 (2013) 056 [arXiv: 1303.0836] [INSPIRE].

[21] D. Xie, M5 brane and four dimensional $N=1$ theories I, JHEP 04 (2014) 154 [arXiv: 1307.5877] [INSPIRE].

[22] I. Bah and N. Bobev, Linear quivers and $\mathcal{N}=1$ SCFTs from M5-branes, JHEP 08 (2014) 121 [arXiv: 1307.7104] [INSPIRE].

[23] P. Agarwal and J. Song, New $N=1$ Dualities from M5-branes and Outer-automorphism Twists, JHEP 03 (2014) 133 [arXiv: 1311.2945] [INSPIRE].

[24] P. Agarwal, I. Bah, K. Maruyoshi and J. Song, Quiver tails and $\mathcal{N}=1$ SCFTs from M5-branes, JHEP 03 (2015) 049 [arXiv: 1409.1908] [INSPIRE].

[25] P. Agarwal, K. Intriligator and J. Song, Infinitely many $\mathcal{N}=1$ dualities from $m+1-m=1$, JHEP 10 (2015) 035 [arXiv: 1505.00255] [INSPIRE].

[26] A. Gadde, E. Pomoni, L. Rastelli and S.S. Razamat, S-duality and 2d Topological QFT, JHEP 03 (2010) 032 [arXiv: 0910.2225] [InSPIRE].

[27] A. Gadde, L. Rastelli, S.S. Razamat and W. Yan, The 4d Superconformal Index from q-deformed $2 d$ Yang-Mills, Phys. Rev. Lett. 106 (2011) 241602 [arXiv:1104.3850] [InSPIRE].

[28] A. Gadde, L. Rastelli, S.S. Razamat and W. Yan, Gauge Theories and Macdonald Polynomials, Commun. Math. Phys. 319 (2013) 147 [arXiv:1110.3740] [inSPIRE].

[29] D. Gaiotto, L. Rastelli and S.S. Razamat, Bootstrapping the superconformal index with surface defects, JHEP 01 (2013) 022 [arXiv:1207.3577] [INSPIRE].

[30] N. Mekareeya, J. Song and Y. Tachikawa, 2d TQFT structure of the superconformal indices with outer-automorphism twists, JHEP 03 (2013) 171 [arXiv:1212.0545] [INSPIRE].

[31] M. Lemos, W. Peelaers and L. Rastelli, The superconformal index of class $S$ theories of type D, JHEP 05 (2014) 120 [arXiv: 1212.1271] [INSPIRE].

[32] C. Beem and A. Gadde, The $N=1$ superconformal index for class $S$ fixed points, JHEP 04 (2014) 036 [arXiv: 1212.1467] [INSPIRE].

[33] Y. Tachikawa, A review of the $T_{N}$ theory and its cousins, PTEP 2015 (2015) $11 \mathrm{~B} 102$ [arXiv: 1504.01481] [INSPIRE].

[34] K.A. Intriligator and B. Wecht, The exact superconformal R symmetry maximizes a, Nucl. Phys. B 667 (2003) 183 [hep-th/0304128] [INSPIRE].

[35] S.M. Kuzenko and S. Theisen, Correlation functions of conserved currents in $N=2$ superconformal theory, Class. Quant. Grav. 17 (2000) 665 [hep-th/9907107] [INSPIRE].

[36] O. Chacaltana, J. Distler and Y. Tachikawa, Nilpotent orbits and codimension-two defects of $6 d N=(2,0)$ theories, Int. J. Mod. Phys. A 28 (2013) 1340006 [arXiv:1203.2930] [INSPIRE].

[37] L.F. Alday, F. Benini and Y. Tachikawa, Liouville/Toda central charges from M5-branes, Phys. Rev. Lett. 105 (2010) 141601 [arXiv:0909.4776] [INSPIRE].

[38] A.D. Shapere and Y. Tachikawa, Central charges of $N=2$ superconformal field theories in four dimensions, JHEP 09 (2008) 109 [arXiv:0804.1957] [INSPIRE]. 
[39] J.A. Harvey, R. Minasian and G.W. Moore, NonAbelian tensor multiplet anomalies, JHEP 09 (1998) 004 [hep-th/9808060] [INSPIRE].

[40] K.A. Intriligator, Anomaly matching and a Hopf-Wess-Zumino term in $6 d, N=(2,0)$ field theories, Nucl. Phys. B 581 (2000) 257 [hep-th/0001205] [INSPIRE].

[41] P. Yi, Anomaly of (2,0) theories, Phys. Rev. D 64 (2001) 106006 [hep-th/0106165] [INSPIRE].

[42] D. Kutasov, A. Parnachev and D.A. Sahakyan, Central charges and $\mathrm{U}(1)_{R}$ symmetries in $N=1$ super Yang-Mills, JHEP 11 (2003) 013 [hep-th/0308071] [INSPIRE].

[43] L. Rastelli and S.S. Razamat, The Superconformal Index of Theories of Class $\mathcal{S}$, in New Dualities of Supersymmetric Gauge Theories, J. Teschner ed., Springer (2015), pg. 261-305 [arXiv: 1412.7131].

[44] D. Gaiotto and S.S. Razamat, $\mathcal{N}=1$ theories of class $\mathcal{S}_{k}, J H E P 07(2015) 073$ [arXiv: 1503.05159] [INSPIRE].

[45] S. Franco, H. Hayashi and A. Uranga, Charting Class $\mathcal{S}_{k}$ Territory, Phys. Rev. D 92 (2015) 045004 [arXiv: 1504.05988] [INSPIRE].

[46] A. Hanany and K. Maruyoshi, Chiral theories of class S, JHEP 12 (2015) 080 [arXiv: 1505. 05053] [INSPIRE].

[47] M. Del Zotto, C. Vafa and D. Xie, Geometric engineering, mirror symmetry and $6 \mathrm{~d}_{(1,0)} \rightarrow 4 \mathrm{~d}_{(\mathcal{N}=2)}$, JHEP 11 (2015) 123 [arXiv: 1504.08348] [INSPIRE].

[48] J.J. Heckman, P. Jefferson, T. Rudelius and C. Vafa, Punctures for Theories of Class $\mathcal{S}_{\Gamma}$, arXiv: 1609.01281 [INSPIRE].

[49] S.S. Razamat, C. Vafa and G. Zafrir, $4 d N=1$ from $6 d(1,0)$, arXiv:1610.09178 [INSPIRE].

[50] P. Putrov, J. Song and W. Yan, (0,4) dualities, JHEP 03 (2016) 185 [arXiv:1505.07110] [INSPIRE].

[51] O. Aharony, N. Seiberg and Y. Tachikawa, Reading between the lines of four-dimensional gauge theories, JHEP 08 (2013) 115 [arXiv:1305.0318] [INSPIRE].

[52] S.S. Razamat and B. Willett, Global Properties of Supersymmetric Theories and the Lens Space, Commun. Math. Phys. 334 (2015) 661 [arXiv:1307.4381] [inSPIRE]. 